\title{
Differential geometry: a natural tool for describing symmetry operations
}

\author{
Philippe Kocian, Kurt Schenk and Gervais Chapuis
}

Acta Cryst. (2009). A65, 329-341

Copyright (C) International Union of Crystallography

Author(s) of this paper may load this reprint on their own web site or institutional repository provided that this cover page is retained. Republication of this article or its storage in electronic databases other than as specified above is not permitted without prior permission in writing from the IUCr.

For further information see http://journals.iucr.org/services/authorrights.html

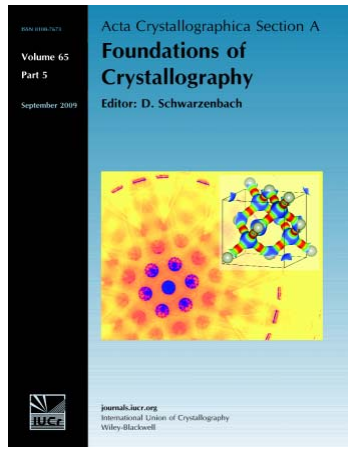

Acta Crystallographica Section A: Foundations of Crystallography covers theoretical and fundamental aspects of the structure of matter. The journal is the prime forum for research in diffraction physics and the theory of crystallographic structure determination by diffraction methods using X-rays, neutrons and electrons. The structures include periodic and aperiodic crystals, and non-periodic disordered materials, and the corresponding Bragg, satellite and diffuse scattering, thermal motion and symmetry aspects. Spatial resolutions range from the subatomic domain in charge-density studies to nanodimensional imperfections such as dislocations and twin walls. The chemistry encompasses metals, alloys, and inorganic, organic and biological materials. Structure prediction and properties such as the theory of phase transformations are also covered.

\section{Crystallography Journals Online is available from journals.iucr.org}


Acta Crystallographica Section A

Foundations of Crystallography

ISSN 0108-7673

Received 3 October 2008

Accepted 26 June 2009

(C) 2009 International Union of Crystallography

Printed in Singapore - all rights reserved

\section{Differential geometry: a natural tool for describing symmetry operations}

\author{
Philippe Kocian, ${ }^{*}$ Kurt Schenk and Gervais Chapuis
}

Laboratoire de Cristallographie, IPMC-FSB, École Polytechnique Fédérale de Lausanne, BSP-Le Cubotron, Dorigny, $\mathrm{CH}-1015$ Lausanne, Switzerland. Correspondence e-mail:

philippe.kocian@epfl.ch

Differential geometry provides a useful mathematical framework for describing the fundamental concepts in crystallography. The notions of point and associated vector spaces correspond to those of manifold and tangent space at a given point. A space-group operation is a one-to-one map acting on the manifold, whereas a point-group operation is a linear map acting between two tangent spaces of the manifold. Manifold theory proves particularly powerful as a unified formalism describing symmetry operations of conventional as well as modulated crystals without requiring a higher-dimensional space. We show, in particular, that a modulated structure recovers a three-dimensional periodicity in any tangent space and that its point group consists of linear applications.

\section{Introduction}

The common mathematical tool for the geometrical description of crystals is that of vector spaces. Our three-dimensional space (in which crystals do exist) is called point space $\mathcal{E}$ and its properties are mainly dictated by those of the so-called associated vector space $V$. This is achieved by associating a vector $P Q \doteqdot r$ of $V$ with any two points $P$ and $Q$ of $\mathcal{E}$. The scalar product of the pre-Hilbert space $V$ then provides lengths between points and angles between lines in $V$.

Excellent textbooks such as those written by W. Opęchowski (1986), H. Burzlaff \& H. Zimmermann (1977), H. Wondratschek (2002), P. Engel (1986) and D. Schwarzenbach (1993) commence by stating that any isometry on the point space may be represented by a unimodular matrix $F$ and a shift vector $s$. Although such an operation $\{F \mid s\}$ is indeed an isometry on a manifold with constant and null curvature, it would appear desirable to furnish a proof that this is the only possible form of isometry in this case. We shall demonstrate (§2.2) that such a proof arises naturally from an analysis of the isometries on a Riemannian manifold. In other instances, the necessity of differential geometry manifests in an even clearer way: e.g. when determining Poincaré's group in special relativity (Das, 1993), even in the simplest case of flat space-time.

As we shall see below, manifold theory is a suitable framework for dealing with geometry. One might think that it is just a complicated way of describing the same things as in the traditional point of view, especially when the space considered is Euclidean. This is definitely not the case, since the traditional point of view does not offer the possibility of studying local features; a property is valid not only in the neighbourhood of a point, but everywhere, which is quite restrictive in a sense. This local aspect is of prime importance in the theory of defects: disclinations, for example, are described in curved, even non-Riemannian, manifolds using exterior calculus, Grassman algebras, fibre bundles and the first homotopy group (Kleman \& Friedel, 2008).

The main difference between the theories of manifolds and vector spaces is that the former treats geometry not only globally, but also locally. The vectors (in the theory of vector spaces) are replaced by vector fields (in manifold theory), even if the field is constant. To each vector corresponds a point in the manifold, namely the base point of this vector. The scalar product is replaced by a metric tensor, which is constant if the manifold is Euclidean. Adopting this point of view is fundamental because only in this manner is it possible to properly treat the notion of isometry.

In any case, as soon as we are dealing with a physical object, we must define an origin point. This can only be done in point space and the ensuing analysis of isometries yields affine transformations, not endomorphisms: the Euclidean space has the structure of a manifold rather than of a vector space. Further, we should consider that two points are linked by a curve (in many instances of minimal length) rather than by a vector. This fact is essential for understanding the behaviour of modulated structures.

The goal of this article is to show how it is possible to easily obtain the general form of an isometry on the Euclidean manifold and how the notions of point-group and space-group operations become natural in the frame of differential geometry. These concepts make sense for all crystals, be they modulated or not; so whenever we say crystal or structure, both cases will be understood.

\section{Riemannian manifolds}

The notion of manifold arises from a generalization of objects like curves and surfaces, the geometrical properties of which 
have already been treated by Gauss and his disciple Riemann. Some pertinent definitions and results from manifold theory, used throughout this article, are summarized in \$2.1. A more advanced treatment may be found in the monograph by $\mathrm{B}$. O’Neill (1983).

\subsection{Manifold and tangent spaces}

A smooth manifold $M$ of dimension $n$ is a Hausdorff (separated) space such that for every point $p \in M$ there exists a homeomorphism $\varphi$ between an open neighbourhood $U \subset M$ containing $p$ and an open neighbourhood $U^{\prime} \subset \mathbb{R}^{n}$. Usually, $M$ can be considered embedded in $\mathbb{R}^{m}, m \geq n$. The couple $(U ; \varphi)$ is called a coordinate system and the couple $\left(U^{\prime} ; \varphi^{-1}\right)$ a (local) parameterization of $M$. If $\left(U_{1} ; \varphi_{1}\right)$ and $\left(U_{2} ; \varphi_{2}\right)$ are two different coordinate systems such that $U_{1} \cap U_{2} \neq \emptyset$, then $\varphi_{2} \circ \varphi_{1}^{-1}: \varphi_{1}\left(U_{1} \cap U_{2}\right) \mapsto \varphi_{2}\left(U_{1} \cap U_{2}\right)$ is called a change of coordinates. If $\varphi_{2} \circ \varphi_{1}^{-1}$ is of class $C^{\infty}$, we say that the change of coordinate is smooth. A family $\mathcal{A}=\left\{\left(U_{i} ; \varphi_{i}\right) \mid i \in J\right\}$ of coordinate systems $(J \subset \mathbb{N})$ is called an atlas of $M$ if $\bigcup_{i \in J} U_{i}=M$. If all changes of coordinates are smooth, we say that the atlas is smooth. In coordinate language: $(U, \varphi)$ is a set of $n$ functions $\varphi=\left(x^{1} ; \ldots ; x^{n}\right), \quad x^{r}: M \mapsto \mathbb{R} \quad$ with $\varphi\left(a^{1} ; \ldots ; a^{m}\right)=\left(x^{1}\left(a^{1} ; \ldots ; a^{m}\right) ; \ldots ; x^{n}\left(a^{1} ; \ldots ; a^{m}\right)\right)=$ $\left(b^{1} ; \ldots ; b^{n}\right) \in \mathbb{R}^{n}, \quad\left(a^{1} ; \ldots ; a^{m}\right) \in M \subset \mathbb{R}^{m}, \quad m \geq n . \quad$ If $x^{r}\left(a^{1} ; \ldots ; a^{m}\right) \doteqdot u^{r}\left(a^{1} ; \ldots ; a^{m}\right)=a^{r}$, then $\varphi\left(a^{1} ; \ldots ; a^{m}\right)$ $=\left(a^{1} ; \ldots ; a^{n}\right)$ and $\varphi=\left(u^{1} ; \ldots ; u^{n}\right)$ is the identity map, also called the natural coordinate system.

The $n$-dimensional Euclidean space $\mathbb{R}^{n}$ itself may be understood as a manifold in which the identity map furnishes a one-coordinate system atlas. The sphere $\mathcal{S}^{2} \subset \mathbb{R}^{3}$ is a twodimensional manifold. Indeed, a point on $\mathcal{S}^{2}$ may be specified by three Cartesian coordinates $\left(a^{1} ; a^{2} ; a^{3}\right) \in \mathbb{R}^{3}$ (with a constraint on them) or by its latitude and longitude $(\theta ; \phi)$. Here we have $m=3, n=2$ and $\varphi=\left(x^{1} ; x^{2}\right)$, with $x^{1}\left(a^{1} ; a^{2} ; a^{3}\right)=\theta \doteqdot b^{1}, \quad x^{2}\left(a^{1} ; a^{2} ; a^{3}\right)=\phi \doteqdot b^{2} . \quad$ Generally speaking, every surface in three-dimensional Euclidean space (e.g. cylinder, torus, paraboloid) is a two-dimensional manifold.

Consider now a curve $\gamma: I \rightarrow M$ on a manifold $M$, where $I \subset \mathbb{R}$ is an interval; let $\mathcal{C}_{p}(M)$ be the set of all curves $(I ; \gamma)$, such that $0 \in I$ and that $\gamma(0)=p$. The subset of $(I ; \gamma)$ in which all curves have the same derivative at 0 forms an equivalence class. Such a class is called a tangent vector at $p$ and is noted $\dot{\gamma}(0)=\left.(\mathrm{d} / \mathrm{d} t) \gamma(t)\right|_{t=0}$; the set of all tangent vectors at $p$ has the structure of a vector space; it is called the tangent space at $p$ and is noted $\mathrm{T}_{p} M$. As an example of a tangent vector, we think of a ship sailing on the sea. At any point $p$ of the sea (on the Earth's surface), the ship's velocity vector is a vector tangent to the Earth at this point.

For every coordinate system $\left(x^{1} ; \ldots ; x^{n}\right)$ on a manifold $M$, the set of derivatives $\left[\left.\left(\partial / \partial x^{1}\right)\right|_{p} ; \ldots ;\left.\left(\partial / \partial x^{n}\right)\right|_{p}\right]$ forms a basis of the tangent space $\mathrm{T}_{p} M$ at $p$; this basis is called the canonical basis of $\mathrm{T}_{p} M$.

We shall write TM for the set of all tangent vectors of a manifold $M$. A vector $v$ belongs to TM if and only if there exists a point $p \in M$ such that $v \in \mathrm{T}_{p} M$. TM is called the tangent bundle of $M$ and can be written as $\mathrm{T} M=\bigcup_{p \in M} \mathrm{~T}_{p} M$. A vector field on an open set $W \subset M$ is a mapping $Y: W \rightarrow \mathrm{T} M$ which assigns a tangent vector $Y_{p} \in \mathrm{T}_{p} M$ to each point $p \in W$.

\subsection{Isometries on a Riemannian manifold}

A metric tensor $g$ on a smooth manifold $M$ is a symmetric, positive definite $(0 ; 2)$, i.e. two times covariant, tensor field on $M$. In other words:

$$
\begin{aligned}
g: \mathrm{T} M \times \mathrm{T} M & \rightarrow \mathbb{R} \\
(v ; w) & \rightarrow g(v ; w) ;
\end{aligned}
$$

$g$ associates each point $p \in M$ with a scalar product $g_{p}$ in the tangent space $\mathrm{T}_{p} M$ at $p$. A manifold $M$ furnished with a metric tensor $g$ is called a Riemannian manifold and is noted $(M ; g)$.

The metric tensor is fundamental because it provides important definitions such as lengths of curves on a manifold and angles between tangent vectors. Moreover, the manifold can be seen as a metric space. Indeed, in every connected manifold we can define the distance between two points $p$ and $q$ as the length of the curve of minimal length belonging to the manifold and linking these two points. In many cases, especially when the manifold has no boundary, this curve is a geodesic.

Let $\phi$ be a map from a Riemannian manifold $(M ; g)$ into itself. We then define an application $\mathrm{d} \phi_{p}: \mathrm{T}_{p} M \rightarrow \mathrm{T}_{\phi(p)} M$ which is called the differential map of $\phi$ at the point $p \in M$. In a coordinate system $x=\left(x^{1} ; \ldots ; x^{n}\right), \mathrm{d} \phi_{p}$ is represented by the Jacobian matrix $\left(\left(\partial x^{\prime i} / \partial x^{j}\right)(p)\right)_{i, j=1}^{n}$, where $x^{\prime}=x \circ \phi$. A map $\phi: M \rightarrow M$ is called a local isometry if for all $p \in M$ and $v, w \in \mathrm{T}_{p} M$

$$
g\left(\mathrm{~d} \phi_{p}(v) ; \mathrm{d} \phi_{p}(w)\right)=g(v ; w) ;
$$

it is an isometry if it is also a diffeomorphism. One might think that this definition is somewhat artificial and specific to manifold theory, but it is altogether consistent with our intuitive notion of isometry. Indeed, consider a (smooth) curve $\gamma$ parameterized between $a$ and $b$; its length is $\int_{a}^{b}[g(\dot{\gamma}(t) ; \dot{\gamma}(t))]^{1 / 2} \mathrm{~d} t$; as $g(\dot{\gamma}(t) ; \dot{\gamma}(t))=$ $g\left(\mathrm{~d} \phi_{\gamma(t)}(\dot{\gamma}(t)) ; \mathrm{d} \phi_{\gamma(t)}(\dot{\gamma}(t))\right)$, we deduce that the length of any curve remains unchanged under an isometry, hence also distances. Using the same considerations, one shows that the angle between two curves crossing at a point $p$ remains unchanged under an isometry of the manifold.

Note that the set of isometries forms a group. In the case where $v=\left.\left(\partial / \partial x^{i}\right)\right|_{p}$ and $w=\left.\left(\partial / \partial x^{j}\right)\right|_{p}$, with respect to the coordinate system $x=\left(x^{1} ; \ldots ; x^{n}\right)$, relation (1) becomes

$$
g_{i j}(x)=\sum_{k, l=1}^{n} \frac{\partial x^{\prime k}}{\partial x^{i}} \frac{\partial x^{\prime l}}{\partial x^{j}} g_{k l}\left(x^{\prime}\right),
$$

where $x^{\prime}=x \circ \phi$.

As the simplest example, let us consider the $n$-dimensional Euclidean space as a Riemannian manifold endowed with the natural coordinate system $u=\left(u^{1} ; \ldots ; u^{n}\right)$. By definition, the metric tensor is constant and represented, in this coordinate system, by the identity matrix $I_{n}$, the components of which are 
$g_{i j}=\delta_{i j}$ for all $1 \leq i, j \leq n$, and $p \in M$. Relation (2) becomes here

$$
\delta_{i j}=\sum_{k, l=1}^{n} \frac{\partial u^{\prime k}}{\partial u^{i}} \frac{\partial u^{\prime l}}{\partial u^{j}} \delta_{k l}
$$

The derivative of this expression with respect to $u^{m}$ is

$$
0=\frac{\partial}{\partial u^{m}} \delta_{i j}=\sum_{k, l=1}^{n}\left(\frac{\partial^{2} u^{\prime k}}{\partial u^{m} \partial u^{i}} \frac{\partial u^{\prime l}}{\partial u^{j}}+\frac{\partial u^{\prime k}}{\partial u^{i}} \frac{\partial^{2} u^{\prime l}}{\partial u^{m} \partial u^{j}}\right) \delta_{k l} .
$$

Cyclic permutation of indices $(m ; i ; j)$ in the formula above yields two similar expressions in $(j ; m ; i)$ and $(i ; j ; m)$. Adding the first two of these and subtracting the third one, we obtain, after some algebra,

$$
\frac{\partial^{2} u^{\prime i}}{\partial u^{m} \partial u^{j}}=0
$$

for all $1 \leq i, j, m \leq n$ and at any point $p \in M$. Integrating this last expression, we find

$$
f_{j}^{i} \doteqdot \frac{\partial u^{\prime i}}{\partial u^{j}}=\text { constant }
$$

If we integrate again, we finally obtain

$$
u^{\prime i}=\sum_{i=1}^{n} f_{j}^{i} u^{j}+s^{i}
$$

where $s^{i}$ is a constant and $f_{j}^{i}$ is the component $(i ; j)$ of a constant matrix $F$, which is, in our case, orthogonal. Indeed, the matrix expression of relation (3) is ${ }^{\mathrm{t}} F F=I_{n}$. In the case in which the coordinate system is not orthogonal (but still 'flat', i.e. not curved), the matrix $F$ is still constant but is characterized by the relation ${ }^{\mathrm{t}} F G F=G$, where $G$ is the (constant) matrix representing the metric tensor in this coordinate system.

\section{Curves versus vectors}

Knowing that in general manifolds differ from vector spaces, the jargon must be adapted to this context. Any two points in a connected manifold can be linked by a curve, but usually not by a vector. For instance, the trajectory of a ship sailing from Port Askaig (Scotland) to Port Fairy (Australia) is not a
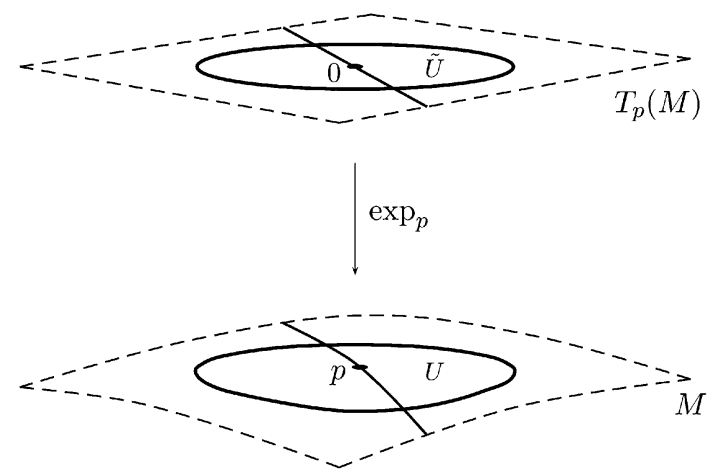

Figure 1

Illustration of the exponential map. It carries lines through the origin of $\mathrm{T}_{p} M$ to geodesics of $M$ through $p$. straight line (the ship is not boring a tunnel through the Earth) but a curve on the surface of our planet (this curve is usually of minimal length when there is no continent or isle between the starting and end points).

In manifold theory, vectors are elements of tangent spaces (which are vector spaces), not of manifolds. The question is why vectors and not curves are always used for linking two points in Euclidean space. To answer this question, the notions of geodesic and exponential map have to be introduced. Briefly, a geodesic on a Riemannian manifold is a curve which can be defined from one of its more important properties, that is a curve of minimal length; for any two points on a connected Riemannian manifold without boundary the curve of minimal length linking these two points is a geodesic. For example, geodesics are straight lines in Euclidean space and arcs of big circles on the sphere $\mathcal{S}^{2}$. Let $c_{v}$ be the maximal geodesic (i.e. it cannot be extended) on a Riemannian manifold $M$ such that $c_{v}(0)=p \in M,\left(\mathrm{~d} c_{v} / \mathrm{d} t\right)(0)=\dot{c}_{v}(0)=v$, and $\widetilde{\mathrm{T}_{p} M}$, the set of all vectors $v$ of $\mathrm{T}_{p} M$ such that $c_{v}$ is defined at least in $[0 ; 1]$. Then, the exponential map of $M$ at $p$ is the function

$$
\begin{aligned}
\exp _{p}: \widetilde{\mathrm{T}_{p} M} & \rightarrow M \\
v & \mapsto \exp _{p}(v)=c_{v}(1)
\end{aligned}
$$

For a fixed $v, \exp _{p}(t v)=c_{t v}(1)=c_{v}(t)$, where $t \in \mathbb{R}$. Thus the exponential map $\exp _{p}$ carries lines through the origin of $\mathrm{T}_{p} M$ to geodesics of $M$ through $p$ (see Fig. 1). Note that for each point $p \in M$, there exists a neighbourhood $\tilde{U}$ of 0 in $\mathrm{T}_{p} M$ on which the exponential map $\exp _{p}$ is a diffeomorphism into a neighbourhood $U$ of $p$ in $M$. In simple cases, as for example in Euclidean space, $\tilde{U}=\mathrm{T}_{p} M$ and $U=M$.

Let $\mathbb{R}^{n}$ be the $n$-dimensional Euclidean space endowed with its natural coordinate system $u=\left(u^{1} ; \ldots ; u^{n}\right)$, in which the components of the metric tensor are $g_{i j}(p)=\delta_{i j}$ for all $1 \leq i, j \leq n$ and for all $p \in \mathbb{R}^{n}$. Recall that geodesics are straight lines in this space. Take two points $q$ and $p$ in $\mathbb{R}^{n}$ with coordinates $u_{q}=\left(u_{q}^{1} ; \ldots ; u_{q}^{n}\right)$ and $u_{p}=\left(u_{p}^{1} ; \ldots ; u_{p}{ }^{n}\right)$, respectively. They are linked by a unique geodesic, a straight line. This geodesic, linearly parameterized between 0 and 1 (i.e. with a constant velocity) is given by

$$
\begin{aligned}
c:[0 ; 1] & \rightarrow \mathbb{R}^{n} \\
t & \mapsto c(t)=u_{q}+\left(u_{p}-u_{q}\right) t
\end{aligned}
$$

with $c(0)=u_{q}$ and $c(1)=u_{p}$; its tangent vector at $q$ is

$$
\left.\frac{\mathrm{d} c(t)}{\mathrm{d} t}\right|_{t=0}=\dot{c}(0)=u_{p}-u_{q} \doteqdot v \text {. }
$$

This vector and the starting point $c(0)=u_{q}$ completely characterize the curve $c$, which will then be noted $c_{v}$; indeed, one can write

$$
c_{v}(t)=u_{q}+v t
$$

and we observe that the end point of $c_{v}$, namely $c_{v}(1)=u_{p}$, can be seen as the tip of the tangent vector of the curve $c_{v}$ at $q$ :

$$
u_{p}=c_{v}(1)=\underbrace{u_{q}}_{\in \mathbb{R}^{n}}+\underbrace{v}_{\in \mathrm{T}_{q} \mathbb{R}^{n}}
$$


In the case where $q$ is the origin point $o$ of the coordinate system [with coordinates $u_{o}=(0 ; \ldots ; 0)=0$ ], we have $u_{p}=c_{v}(1)=u_{o}+v=v$, which can be written $\operatorname{asp}_{o}(v)=v$. Even if this expression is perfectly correct, it must be noted that $c_{v}(1)$ is a point of $\mathbb{R}^{n}$ whereas $v$ is an element of $\mathrm{T}_{o} \mathbb{R}^{n}$. This result is fundamental because it shows that $\mathrm{T}_{o} \mathbb{R}^{n}$ and $\mathbb{R}^{n}$ are not only isomorphic but also geometrically equivalent, that is isometric (also note that the metric tensor, the components of which in the natural coordinate system are $\delta_{i j}, 1 \leq i, j \leq n$, is constant and equal to the scalar product it generates in the tangent space).

Let us consider an $n$-dimensional periodic lattice $\Lambda$ in the Euclidean space $\mathbb{R}^{n}$; it can be written as

$$
\Lambda=\left\{B \lambda \mid \lambda=\left(\lambda_{1} ; \ldots ; \lambda_{n}\right) \in \mathbb{Z}^{n}\right\},
$$

where the invertible matrix $B\left[\in \mathrm{GL}_{n}(\mathbb{R})\right]$ defines the geometry of the lattice. The geodesic (straight line) linking the origin $o$ [with coordinates $u_{o}=(0 ; \ldots ; 0)=0$ ] to any node of $\Lambda$, and linearly parameterized between 0 and 1 , may be written as

$$
\begin{aligned}
c:[0 ; 1] & \rightarrow \mathbb{R}^{n} \\
t & \mapsto c(t)=B \lambda t .
\end{aligned}
$$

The tangent vector of this geodesic at the origin is

$$
\dot{c}(0)=B \lambda \text {. }
$$
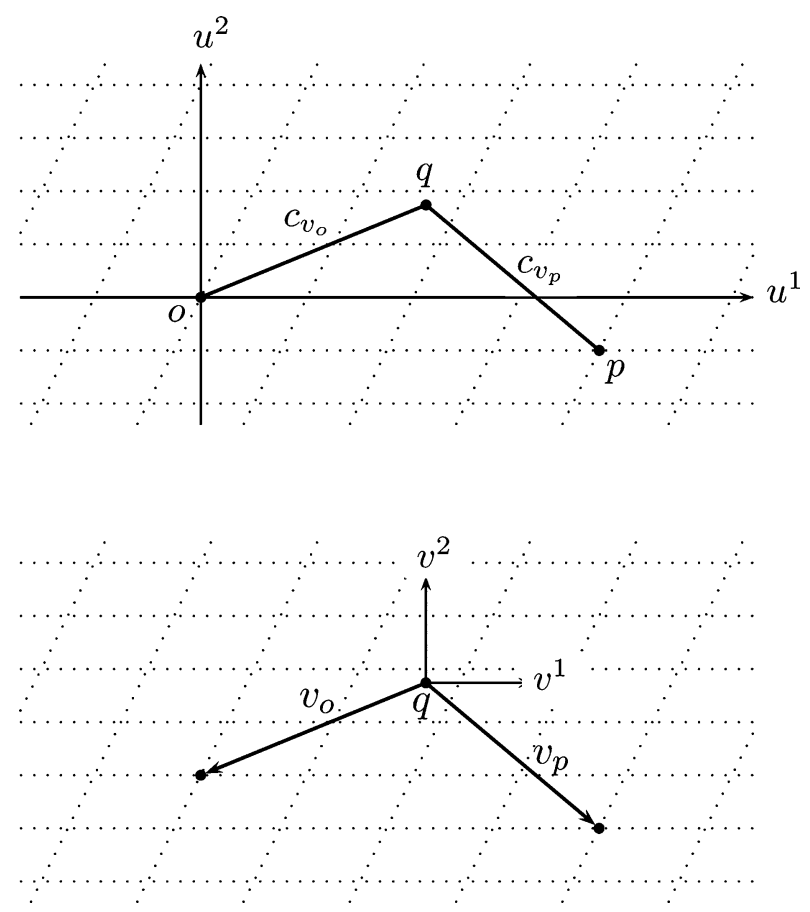

Figure 2

Example of a two-dimensional periodic lattice viewed in the manifold $\mathbb{R}^{2}$ endowed with the natural Cartesian coordinate system and in its tangent space at $q, \mathrm{~T}_{q} \mathbb{R}^{2}$. Note that we could also use the appropriate crystallographic coordinate system (with respect to the lattice parameters); the result would be exactly the same.
If we take the tangent vector at the origin of all geodesics linking the origin to each node of $\Lambda$, we obtain the following set of vectors:

$$
\Upsilon_{o}=\left\{B \lambda \mid \lambda \in \mathbb{Z}^{n}\right\}
$$

which shall be called the representation of the lattice $\Lambda$ in $\mathrm{T}_{o} \mathbb{R}^{n}$. As the metric tensor is constant on $\mathbb{R}^{n}$ and equal to the scalar product it generates in the tangent space at the origin, we can conclude that the representation $\Upsilon_{o}$ of the lattice $\Lambda$ in $\mathrm{T}_{o} \mathbb{R}^{n}$ is equal to the lattice $\Lambda$ itself.

Let us also try to find the representation of this same lattice $\Lambda$ in the tangent space of $\mathbb{R}^{n}$ at any point $q$. Any geodesic linearly parameterized between 0 and 1 , linking the point $q$ of coordinates $u_{q}=\left(u_{q}^{1} ; \ldots ; u_{q}^{n}\right)$ to any node of $\Lambda$, can be written as

$$
\begin{aligned}
c:[0 ; 1] & \rightarrow \mathbb{R}^{n} \\
t & \mapsto c(t)=u_{q}+\left(B \lambda-u_{q}\right) t .
\end{aligned}
$$

Its tangent vector at $q$ is

$$
\dot{c}(0)=B \lambda-u_{q} .
$$

This last expression shows that the representation $\Upsilon_{q}$ of the lattice $\Lambda$ in the tangent space $T_{q} \mathbb{R}^{n}$ shares most features with $\Lambda$ : the pattern is completely the same, it is just translated by $-u_{q}$ (see Fig. 2). This result is in fact completely intuitive: the translation by $-u_{q}$ compensates for the fact that $\mathrm{T}_{q} \mathbb{R}^{n}$ is shifted from the tangent space at the origin of $u_{q}$.

At this point, one might think that this derivation brings nothing new, but is just a more complicated way of describing known things. These considerations are, however, necessary in order to treat structures in a unique way. In modulated structures, atomic positions are those of a basic (or average) structure (with a three-dimensional space-group symmetry) on which a periodic wavefunction of the position is applied (Janssen et al., 2002, 2007; Steurer \& Haibach, 2001; van Smaalen, 2007, 1995). More generally, a modulation is a oneto-one map $H$ from $\mathbb{R}^{n}$ to $\mathbb{R}^{n}$. For crystallographic applications, $H$ is a periodic wavefunction of the position, smooth or piecewise smooth (in the case of crenel or sawtooth functions). We can then look at $\mathbb{R}^{n}$ as an $n$-dimensional manifold $M$ parameterized in a non-Euclidean way; $M$ is included in $\mathbb{R}^{n}$ and $\mathbb{R}^{n}$ is included in $M ; H^{-1}$ is a curved coordinate system. Since $M \subset \mathbb{R}^{n}$, any point $p$ of $M$ is also a point in $\mathbb{R}^{n}$ and can therefore be characterized by the $n$ natural coordinates in $\mathbb{R}^{n}$. To illustrate this, let us consider the sphere $\mathcal{S}^{2} \in \mathbb{R}^{3}$; any point $p$ on the sphere can be characterized by two parameters $\theta_{p}$ and $\varphi_{p}$; it is also a point in $\mathbb{R}^{3}$ with (natural) coordinates $u_{p}^{1}=\sin \theta_{p} \cos \varphi_{p}, u_{p}^{2}=\sin \theta_{p} \sin \varphi_{p}$ and $u_{p}^{3}=\cos \theta_{p}$. Let $H(q)$ and $H(p)$ be any two points of $M$ with (natural) coordinates (in $\left.\mathbb{R}^{n}\right) \quad H\left(u_{q}\right)=\left(h^{1}\left(u_{q}\right) ; \ldots ; h^{n}\left(u_{q}\right)\right) \quad$ and $H\left(u_{p}\right)=\left(h^{1}\left(u_{p}\right) ; \ldots ; h^{n}\left(u_{p}\right)\right)$, respectively. They may be linked in $M$ by a curve parameterized between 0 and 1 as follows:

$$
\begin{aligned}
c:[0 ; 1] & \rightarrow \mathbb{R}^{n} \\
t & \mapsto c(t)=H\left(u_{q}+\left(u_{p}-u_{q}\right) t\right) .
\end{aligned}
$$


Its tangent vector at $H(q)$ is

$$
\left.\frac{\mathrm{d} c(t)}{\mathrm{d} t}\right|_{t=0}=\dot{c}(0)=\mathrm{d} H_{q}\left(u_{p}-u_{q}\right) \doteqdot v ;
$$

in components:

$$
v^{i}=\left.\sum_{j=1}^{n} \frac{\partial h^{i}(u)}{\partial u^{j}}\right|_{q}\left(u_{p}^{j}-u_{q}^{j}\right)
$$

the point $H(p)$ is represented in $\mathrm{T}_{q} M$ by the tip of the arrow $v$.

Note that the curve $\gamma$, which is in $M$, therefore also in $\mathbb{R}^{n}$, is not a straight line, hence not a geodesic. This does not create a problem; the important point is that there exists exactly one curve of the form of $\gamma$ linking the two points $q$ and $p$; this is the case, as $H$ is one-to-one and as there exists exactly one straight line linking two points in $\mathbb{R}^{n} \cdot \gamma$ is the deformation of a geodesic in Euclidean space, through $H$; it is the unique curve
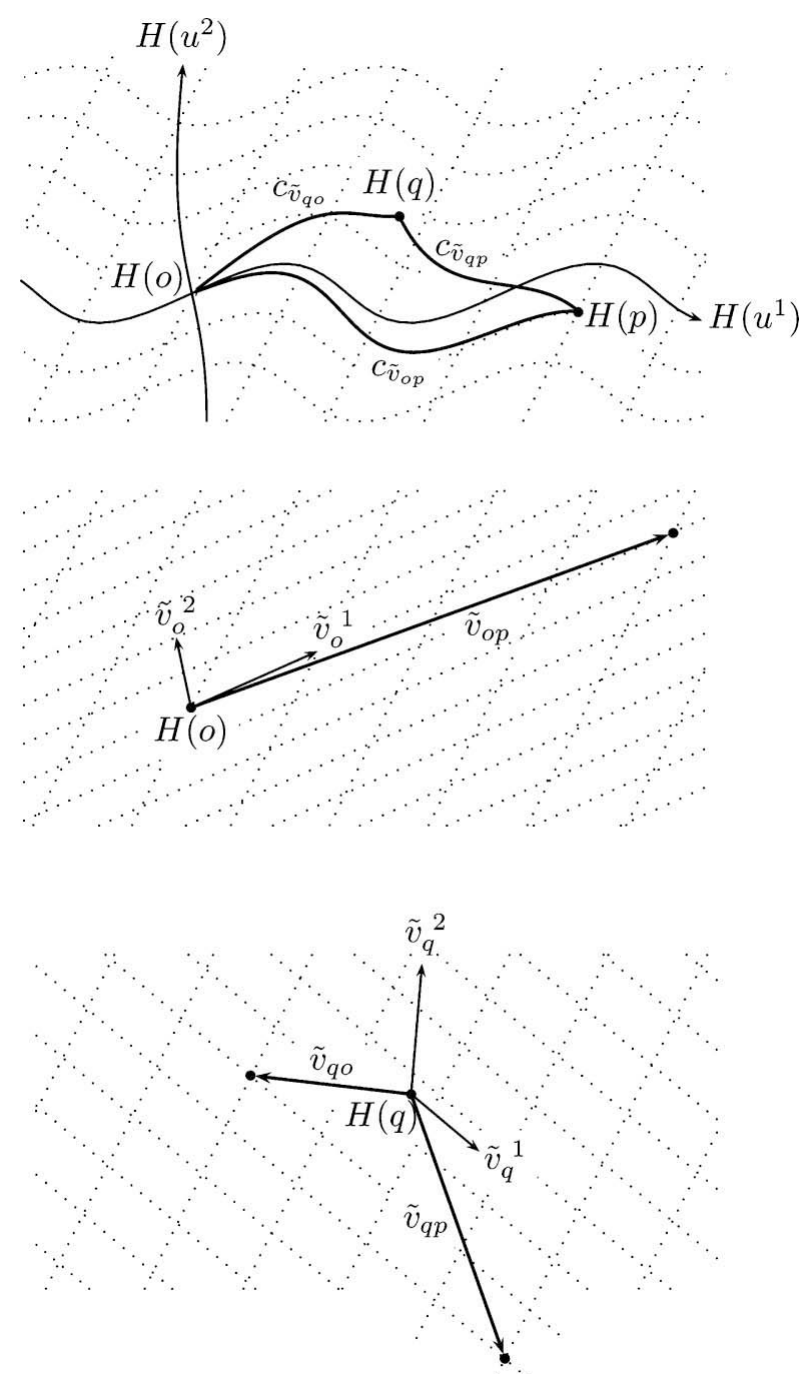

Figure 3

Example of a two-dimensional smooth modulated lattice. The top picture was obtained by taking the previous example and applying the map $H$, which is given by $\left(u^{1} ; u^{2}\right) \mapsto\left(u^{1}+A \sin \left(k u^{1}-a u^{2}\right)\right.$; $\left.u^{2}+B \sin \left(k u^{1}-a u^{2}\right)\right)$. The middle and bottom pictures show the representation of the modulated lattice in the tangent spaces at $H(o)$ and $H(q)$, respectively. In these spaces the lattice has a normal periodicity. starting at $q$, generated by the curve $t \mapsto I_{n} \exp \left(\sum_{j=1}^{n} v^{j} \partial_{j}\right)$, $t \in[0 ; 1]$. Thus, the ideas used in the definition of the exponential map may be generalized to other families of curves.

Let us consider the example of an $n$-dimensional modulated lattice

$$
\tilde{\Lambda}=\left\{H(B \lambda) \mid \lambda \in \mathbb{Z}^{n}\right\},
$$

where $B \in \mathrm{GL}_{n}(\mathbb{R})$ and $H: \mathbb{R}^{n} \rightarrow \mathbb{R}^{n}$ is a one-to-one map, and find its representation in any tangent space. Any point of $\tilde{\Lambda}$ may be linked to $H(o)$, the chosen origin of $M$, by a curve, parameterized between 0 and 1 , which is a deformation through $H$ of a straight line linking the origin $o$ to the corresponding point in the undistorted lattice $\Lambda$ :

$$
\begin{aligned}
c:[0 ; 1] & \rightarrow \mathbb{R}^{n} \\
t & \mapsto c(t)=H(B \lambda t) .
\end{aligned}
$$

Its tangent vector at $H(o)$ is

$$
\dot{c}(0)=\mathrm{d} H_{o}(B \lambda) \doteqdot v_{\lambda} ;
$$

in components:

$$
v_{\lambda}^{i}=\left.\sum_{j=1}^{n} \frac{\partial h^{i}(u)}{\partial u^{j}}\right|_{o}(B \lambda)^{j} .
$$

Thus, the representation of $\tilde{\Lambda}$ in $\mathrm{T}_{H(o)} M$ is the set

$$
\tilde{\Upsilon}_{H(o)}=\left\{\Omega(0) B \lambda \mid \lambda \in \mathbb{Z}^{n}\right\}
$$

where $\Omega(0) \doteqdot\left(\left(\partial h^{i} / \partial u^{j}\right)(0)\right)_{i, j=1}^{n}$. This is a perfectly periodic lattice of translations, in the sense that it has the structure of a finite free $\mathbb{Z}$-module; it corresponds to a linear deformation of $\Lambda$ through the matrix $\Omega(0)$.

If we proceed as before, just replacing $H(0)$ by any point of $M$, for instance $H(q)$, we obtain the curve

$$
\begin{aligned}
c:[0 ; 1] & \rightarrow \mathbb{R}^{n} \\
t & \rightarrow c(t)=H\left(u_{q}+\left(B \lambda-u_{q}\right) t\right),
\end{aligned}
$$

the tangent vector of which at $H(q)$ is

$$
\dot{c}(0)=\mathrm{d} H_{q}\left(B \lambda-u_{q}\right) \doteqdot v_{\lambda}
$$

in components:

$$
v_{\lambda}^{i}=\left.\sum_{j=1}^{n} \frac{\partial h^{i}(u)}{\partial u^{j}}\right|_{q}\left(B \lambda-u_{q}\right)^{j} .
$$

Thus we see that the representation of $\tilde{\Lambda}$ in any tangent space $\mathrm{T}_{H(q)} M$ of $M$ is a periodic lattice, i.e. a finite free $\mathbb{Z}$-module, which can be written as

$$
\tilde{\Upsilon}_{H(q)}=\left\{\Omega\left(u_{q}\right)\left(B \lambda-u_{q}\right) \mid \lambda \in \mathbb{Z}^{n}\right\}
$$

where $\Omega\left(u_{q}\right) \doteqdot\left(\left(\partial h^{i} / \partial u^{j}\right)\left(u_{q}\right)\right)_{i, j=1}^{n}$. It corresponds to the lattice $\Lambda$ distorted by the matrix $\Omega\left(u_{q}\right)$ and eventually translated. Note that this result holds for any one-to-one map $H$, periodic or not; the only condition being that $H$ be smooth on the points where we differentiate it (see Fig. 3).

We can see that the viewpoint just presented provides a common playground for crystals. By designing tools and spaces for this task, the notion of aperiodicity in modulated 
structures disappears. As mentioned previously, vectors are elements of vector spaces and not of manifolds; to link two points on a manifold one uses a curve and not a vector; vectors are used in tangent spaces. In the Euclidean case, curves are straight lines (geodesics) and the end point of such a curve can be seen as the tip of a tangent vector at the starting point of the curve; manifold and tangent space at any point have therefore very close geometrical properties, especially when the tangent point is at the origin (of the coordinate system), in which case these properties completely merge. What is usually called point space is in fact just the Euclidean space seen as a manifold (endowed with the natural coordinate system), while the associated vector space is any tangent space of this manifold (at a selected point). In a crystal, atomic positions are normally described in point space, in the manifold. But as each of these atoms can be seen as an end point of a straight line starting from the origin, therefore as the tip of a tangent vector of the tangent space at the origin, the positions of these atoms might just as well be described in the tangent space at the origin. Often, this second description is used. But are those using it conscious of the fact that they are indeed working in the vector space (tangent space at the origin) and not in point space? The confusion between the Euclidean manifold and its tangent space at the origin arises almost naturally, and it is perhaps the reason why vectors are used to describe the positions of atoms in modulated structures, and why these structures are said to be aperiodic. Consequently, we can draw the following conclusion: modulated crystals are not aperiodic, they have normal vector periodicity in any tangent space, and curve periodicity in the manifold.

\section{Unified description of symmetry operations}

A unified description of crystals is not fully consistent as long as a common formalism for symmetry operations is not obtained. The notion of a symmetry operation of a structure has already been tackled in $\S 3$, when introducing the basic definition of a modulated structure. However, a clear definition is essential. This will be done after discussing the concept of the action of the Euclidean isometry group on a manifold $M$ parameterized by a one-to-one map $H: \mathbb{R}^{n} \rightarrow M$.

\subsection{Euclidean case}

Just as in the theory of vector spaces a linear isometry acts not only on the tip of the arrow but on the whole vector, it is here necessary to study how an isometry acts on whole curves and not on points only. Recall that a straight line (geodesic), linearly parameterized between 0 and 1 , and linking two points $q$ and $p$, with coordinates $u_{q}=\left(u_{q}{ }^{1} ; \ldots ; u_{q}{ }^{n}\right)$ and $u_{p}=\left(u_{p}^{1} ; \ldots ; u_{p}^{n}\right)$, respectively, may be written as

$$
\begin{aligned}
c:[0 ; 1] & \rightarrow \mathbb{R}^{n} \\
t & \mapsto c(t)=u_{q}+\left(u_{p}-u_{q}\right) t ;
\end{aligned}
$$

its tangent vector at $q$ is $v \doteqdot u_{p}-u_{q}$. Let us take an isometry $\phi$ in $\mathbb{R}^{n}$. As established in $\$ 2.2$, it transforms any point of coor- dinates $u$ into another one, of coordinates $u^{\prime}$, according to the relation

$$
u^{\prime}=F u+s, \quad F \in \mathrm{O}_{n}(\mathbb{R}) \text { and } s \in \mathbb{R}^{n} .
$$

Any point of the curve, therefore the whole curve, will then become

$$
c^{\prime}(t)=F c(t)+s=F\left(u_{q}+\left(u_{p}-u_{q}\right) t\right)+s .
$$

The starting point of $c^{\prime}$ is $q^{\prime}$, with coordinates $c^{\prime}(0)=F u_{q}+s$, and the end point is $p^{\prime}$ with coordinates $c^{\prime}(1)=F u_{p}+s$. The tangent vector of $c^{\prime}$ at $q^{\prime}$ is

$$
\left.\frac{\mathrm{d} c^{\prime}(t)}{\mathrm{d} t}\right|_{t=0}=\dot{c}^{\prime}(0)=F\left(u_{p}-u_{q}\right)=F v \doteqdot v^{\prime} .
$$

Let us take some time to interpret these calculations. Note first that $F$ is the matrix representation of $\mathrm{d} \phi_{q}$, the differential map of $\phi$ at $q$ (in fact $F=\mathrm{d} \phi$ at any point). Then recall that the differential map $\mathrm{d} \phi$ carries vectors of the tangent space at a point, let us say $q$, to vectors of the tangent space at the point $\phi(q)$ :

$$
\begin{aligned}
\mathrm{d} \phi_{q}: \mathrm{T}_{q} \mathbb{R}^{n} & \rightarrow \mathrm{T}_{\phi(q)} \mathbb{R}^{n} \\
v & \mapsto \mathrm{d} \phi_{q}(v)=v^{\prime} .
\end{aligned}
$$

Thus, we conclude that there are two ways to determine the image of a point $p$ through an isometry $\phi$. The first, traditional, one consists of applying the matrix and translation parts to $u_{p}$, the coordinates of $p$ :

$$
u_{p}^{\prime}=F u_{p}+s .
$$

The second one consists of considering the point $p$ as the tip of a tangent vector $v$ at $q$ (it is then an element of the tangent space at $q$ ) and applying the differential map $\mathrm{d} \phi_{q}$ of $\phi$ to $v$ in order to obtain the vector $v^{\prime}$ in the tangent space at $\phi(q)$, the tip of which corresponds to a point in $\mathbb{R}^{n}$, namely $\phi(p)$. This alternative way is in fact just a consequence of the Taylor development of $\phi$. Indeed, as $\mathrm{d} \phi$ corresponds, in the natural coordinate system, to a constant matrix $F$ (independent of the point in the manifold), its differential map $\mathrm{d}(\mathrm{d} \phi)$ vanishes everywhere. We have

$$
u_{p}=u_{q}+v, \quad v=\left(u_{p}-u_{q}\right)
$$

and

$$
\phi\left(u_{p}\right)=\phi\left(u_{q}+v\right)=\phi\left(u_{q}\right)+\mathrm{d} \phi_{q}(v) .
$$

In the general case, the left part of equation (13) is a good approximation to the right side if $v$ is small; but the larger $v$ is, the larger the rest of the higher-order terms are. In our case, as $\phi$ corresponds to an affine map, there is no order higher than one and equation (13) holds for every $v$. [Just note that we used the same letter $\phi$ for the isometry and for its representation in coordinates, which is not disturbing in this context.]

One might wonder why such an elaborate procedure is needed to find the image of a point. For one point only, it is indeed needless to do so. But by taking a large number of points, for instance nodes on an infinite lattice of translations [see expression (9)], it becomes immediately more useful. 
Indeed, considering one point $q$ as an origin point and using the technique presented in $\$ 3$ (i.e. we link all nodes to $q$ by straight lines linearly parameterized between 0 and 1 , and calculate the tangent vectors $v_{q, B \lambda}$ of all these curves at $q$ ), we obtain the representation of this lattice in the tangent space $\mathrm{T}_{q} \mathbb{R}^{n}$ at $q$. The image of all these nodes through a symmetry operation $\phi$ of the lattice can be obtained by calculating the image $\phi(q)$ of the point $q$ and then finding the image of the vectors $v_{q, B \lambda}$ through the differential map $\mathrm{d} \phi_{q}$ (which is linear). This means that the translation part of the symmetry operation appears only in the transformation of one point, the point $q$. The image of all others, the nodes in our case, are given by using the linear map $\mathrm{d} \phi_{q}$. All this is summarized in the expression $\mathrm{d} \phi_{q}: \mathrm{T}_{q} \mathbb{R}^{n} \rightarrow \mathrm{T}_{\phi(q)} \mathbb{R}^{n}$.

(1)

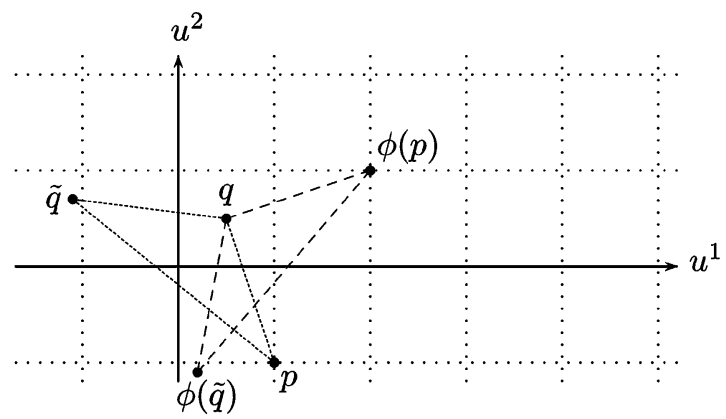

(2)

$\left(2^{\prime}\right)$

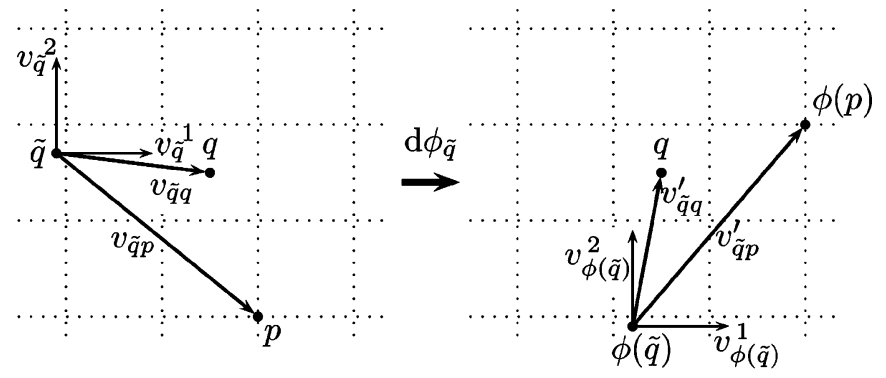

(3)

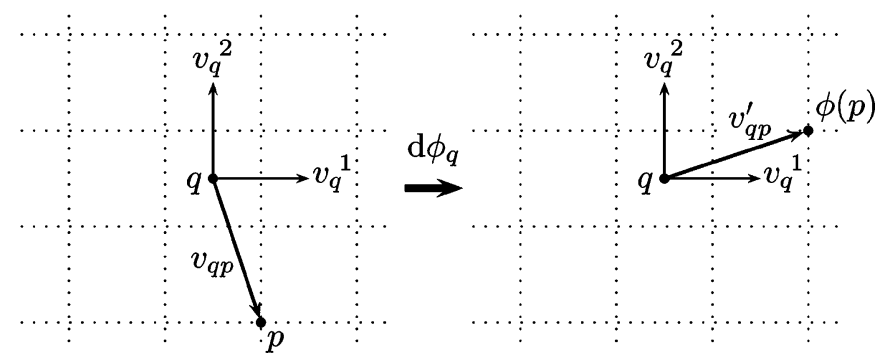

Figure 4

Representations of a symmetry operation in a two-dimensional square lattice. (1) shows the manifold representation of the fourfold rotation $\phi$ on the manifold. Two isometric triangles are drawn, the first one is composed of the points $q, \tilde{q}$ and $p$, the second one of their image. (2) and $\left(2^{\prime}\right)$ show the tangent-space representation of the fourfold rotation. The 'origin point' $\tilde{q}$ (which could also be the real origin point $o$ ) is carried to the 'new origin point' $\phi(\tilde{q})$. The vectors $v_{\tilde{q} p}$ and $v_{\tilde{q} q}$ are carried to $v_{\tilde{q} p}^{\prime}$ and $v_{\tilde{q} q}^{\prime}$, respectively, through the differential map d $\phi_{\tilde{q}}$. In (3) and (3'), we can observe how convenient it is to chose the 'origin point' $\tilde{q}$ at the fixed point $q$; to find the image of $p$, we only need to apply the tangent application $\mathrm{d} \phi_{q}$ to the vector $v_{q p}$.
As an example, we consider the two-dimensional square lattice $\Lambda^{\square}=\left\{a \lambda \mid \lambda \in \mathbb{Z}^{2}\right\}$, where $a \in \mathbb{R}_{+}$. It has, for example, a fourfold point $q$ of coordinates $(a / 2 ; a / 2)$. A rotation $\phi$ of $\pi / 2$ around $q$ may be written, in coordinates, as

$$
\left(\begin{array}{l}
u^{\prime 1} \\
u^{\prime 2}
\end{array}\right)=\left(\begin{array}{cc}
0 & -1 \\
1 & 0
\end{array}\right)\left(\begin{array}{l}
u^{1} \\
u^{2}
\end{array}\right)+\left(\begin{array}{l}
a \\
0
\end{array}\right)
$$

A node $\left(a \lambda^{1} ; a \lambda^{2}\right)$ of this lattice becomes $\left(a\left(-\lambda^{2}+1\right) ; a \lambda^{1}\right)$. Let $\tilde{q}$ be an 'origin point', with coordinates $\left(u_{\tilde{q}}^{1} ; u_{\tilde{q}}^{2}\right)$. Any node of the lattice can be represented in the tangent space at $\tilde{q}$ by taking the tangent vector at $\tilde{q}$ of straight lines, linearly parameterized between 0 and 1 , linking $\tilde{q}$ to the node considered. We obtain

$$
v=\left(\begin{array}{c}
a \lambda^{1}-u_{\tilde{q}}^{1} \\
a \lambda^{2}-u_{\tilde{q}}^{2}
\end{array}\right) .
$$

If we apply the rotation around $q$ to the point $\tilde{q}$, we obtain its image $\phi(\tilde{q})$ with coordinates $\left(u_{\tilde{q}}^{\prime 1} ; u_{\tilde{q}}^{\prime 2}\right)=\left(-u_{\tilde{q}}^{2}+a ; u_{\tilde{q}}^{1}\right)$. This image corresponds to a new 'origin point'. Applying now the matrix part of the rotation $\phi$ to the vectors $v$, we obtain their image:

$$
v^{\prime}=\left(\begin{array}{c}
-a \lambda^{2}+u_{\tilde{q}}^{2} \\
a \lambda^{1}-u_{\tilde{q}}^{1}
\end{array}\right)
$$

which are elements of $\mathrm{T}_{\phi(\tilde{q})} \mathbb{R}^{n}$. These image vectors can be seen as tangent vectors at $\phi(\tilde{q})$ of straight lines, linearly parameterized between 0 and 1 . The end points of these lines correspond to the tip of those tangent vectors. This means that by taking the coordinates of the point $\phi(\tilde{q})$ and adding the components of $v^{\prime}$, the coordinates of the image through $\phi$ of the lattice nodes are obtained; we have

$$
\left(\begin{array}{c}
-u_{\tilde{q}}^{2}+a \\
u_{\tilde{q}}^{1}
\end{array}\right)+\left(\begin{array}{c}
-a \lambda^{2}+u_{\tilde{q}}^{2} \\
a \lambda^{1}-u_{\tilde{q}}^{1}
\end{array}\right)=\left(\begin{array}{c}
a\left(-\lambda^{2}+1\right) \\
a \lambda^{1}
\end{array}\right),
$$

which is exactly the same result as if $\phi$ were applied directly to any node in the manifold. Note that the 'origin point' $\tilde{q}$ can be any point of the manifold, in particular also the 'real origin point' $o$ of the coordinate system; $\phi$ then carries $o$ to $\phi(o)$, the new 'origin point' (see Fig. 4).

We then reach the interesting conclusion that a symmetry operation (in Euclidean space) has two different representations, one in the manifold and another one in the tangent spaces. Moreover, these representations correspond to concepts very well known in crystallography: space-group operation and point-group operation (Wondratschek, 2002). Indeed, a space-group operation is an affine transformation of a point space: this is nothing but an isometry on the Euclidean manifold. A point-group operation is a linear map: this is nothing but an isometry between two tangent spaces. The main difference between the point of view of differential geometry and the traditional one certainly lies in the fact that not only one (associated) vector space but an infinity are considered; at each point of the manifold there exists a tangent space. Then, a point-group operation is in general not an endomorphism of a vector space but a linear map between two tangent spaces. This means, in particular, that the translation 
part of a symmetry operation does not completely vanish: it appears in the image of the 'origin point', i.e. in the fact that vectors of a tangent space are carried to vectors of another tangent space. Realizing this, we can use the manifold representation as well as that in tangent space. Both contain the same information; it is just presented in a different way, whereas in the traditional point of view the information about translation completely disappears when the point-group operation of an isometry is considered. This is due to the fact that only one vector space is considered and no advantage is gained from local geometry.

Note that from the point of view of differential geometry, there are special cases where a point-group operation, that is the differential map of an isometry $\phi$, is an endomorphism, i.e. a linear map from a tangent space into itself. This happens when the isometry has a fixed point $q$. Considering the tangent space at this point and interpreting all other points of the Euclidean manifold as the tips of tangent vectors at $q$, we find that the differential map $\mathrm{d} \phi_{q}$ carries vectors of the tangent space at $q$ to vectors of the tangent space at $\phi(q)=q$ $\left(\mathrm{d} \phi_{q}: \mathrm{T}_{q} \mathbb{R}^{n} \rightarrow \mathrm{T}_{q} \mathbb{R}^{n}\right)$. In the example presented above, we effectively observe that in the tangent space at the rotation point $q$, a node of coordinates $\left(\lambda^{1} ; \lambda^{2}\right)$ is given by the tip of the vector $\left(a\left(\lambda^{1}-\frac{1}{2}\right) ; a\left(\lambda^{2}-\frac{1}{2}\right)\right)$ in $\mathrm{T}_{q} \mathbb{R}^{n}$. Applying then the matrix part (the differential map) of $\phi$, we obtain the vector $\left(-a\left(\lambda^{2}-\frac{1}{2}\right) ; a\left(\lambda^{1}-\frac{1}{2}\right)\right)$, also in $\mathrm{T}_{q} \mathbb{R}^{n}$, which corresponds, in the manifold, to the point $\left(a\left(-\lambda^{2}+1\right) ; a \lambda^{1}\right)$.

\subsection{The modulated case}

Modulated structures are characterized by a periodic deformation of a basic structure, which has normal Euclidean space-group symmetry (Janssen et al., 2002, 2007; Steurer \& Haibach, 2001; van Smaalen, 2007, 1995). In mathematical language, an advantageous way of describing the symmetry of such structures consists of using the action of Euclidean space groups on a manifold [the basic definitions of action of groups on manifolds can be found in the monograph by B. O'Neill (1983)]. In fact, we have already used this concept in the Euclidean case, where the action of isometries was represented by a matrix and a translation part. For modulated structures, this is somewhat more complicated owing to the periodic deformation of the basic structure. Indeed, for a normal periodic structure $\mathcal{S}$, which has a symmetry operation $\phi$, the corresponding modulated structure $H(\mathcal{S})$, where $H: \mathbb{R}^{n} \rightarrow \mathbb{R}^{n}$ is a one-to-one map, has a similar symmetry operation $\tilde{\phi}$, which differs from $\phi$ in that it is sandwiched between $H$ and $H^{-1}$ :

$$
\tilde{\phi}=H \circ \phi \circ H^{-1} \text {. }
$$

Then, as $\phi(\mathcal{S})=\mathcal{S}$, we have

$$
\left(H \circ \phi \circ H^{-1}\right)(H(\mathcal{S}))=H(\phi(\mathcal{S}))=H(\mathcal{S}) .
$$

As in the Euclidean case, let us see how such an operation acts on curves. Recall that two points $H(q)$ and $H(p)$ of a manifold $M$ (parameterized by $H$ ), with coordinates $H\left(u_{q}\right)$ and $H\left(u_{p}\right)$, respectively, may be linked by a curve, parameterized between 0 and 1 , in the following manner:

$$
\begin{aligned}
c:[0 ; 1] & \rightarrow \mathbb{R}^{n} \\
t & \mapsto c(t)=H\left(u_{q}+\left(u_{p}-u_{q}\right) t\right) ;
\end{aligned}
$$

its tangent vector at $H(q)$ is $v \doteqdot \mathrm{d} H_{q}\left(u_{p}-u_{q}\right)$. Take now a Euclidean isometry $\phi$, which can be written as a matrix $F$ and translational part $s$, and sandwich it between $H$ and $H^{-1}$. Any point of the curve, therefore the whole curve, will become under this transformation

$$
c^{\prime}(t)=H\left(\phi\left(u_{q}+\left(u_{p}-u_{q}\right) t\right)\right) .
$$

The starting point of $c^{\prime}$ is $H\left(q^{\prime}\right)$, with coordinates $c^{\prime}(0)=H\left(\phi\left(u_{q}\right)\right)$, and the end point is $H\left(p^{\prime}\right)$, with coordinates $c^{\prime}(1)=H\left(\phi\left(u_{p}\right)\right)$. The tangent vector of $c^{\prime}$ at $H\left(q^{\prime}\right)$ is

$$
\left.\frac{\mathrm{d} c^{\prime}(t)}{\mathrm{d} t}\right|_{t=0}=\dot{c}^{\prime}(0)=\mathrm{d} H_{\phi(q)}\left(\mathrm{d} \phi_{q}\left(u_{p}-u_{q}\right)\right) \doteqdot v^{\prime} .
$$

As $\left(u_{p}-u_{q}\right)=\left(\mathrm{d} H_{q}\right)^{-1}(v)$, the previous expression becomes, using matrix notation,

$$
\dot{c}^{\prime}(0)=v^{\prime}=\Omega(\phi(q)) F \Omega(q)^{-1} v,
$$

where $\Omega$ and $F$ are the matrix representations of $\mathrm{d} H$ and $\mathrm{d} \phi_{q}$, respectively. As in the Euclidean case, we notice that there are two ways of writing a symmetry operation, i.e. how to obtain the image of a point $p$. The first one consists of the application of $\tilde{\phi} \doteqdot H \circ \phi \circ H^{-1}$ to the point $H(p)$, with coordinates $H\left(u_{p}\right)$ :

$$
H\left(u_{p}\right) \mapsto \tilde{\phi}\left(H\left(u_{p}\right)\right)=H\left(\phi\left(u_{p}\right)\right) .
$$

In the second one, we first find the vector in the tangent space at $H(q)$, which corresponds to the tangent vector at $H(q)$ of the curve $c$ (parameterized between 0 and 1) linking $H(q)$ to $H(p)$. Then we apply the linear map $\Omega(\phi(q)) F \Omega(q)^{-1}$, and we finally obtain a vector in the tangent space at $H\left(q^{\prime}\right)$, which is the tangent vector at $H\left(q^{\prime}\right)$ of the curve $c^{\prime}$ (parameterized between 0 and 1 in the same way) linking $H\left(q^{\prime}\right)$ to $H\left(p^{\prime}\right)$. Note that the correspondence between curves (parameterized between 0 and 1) and their tangent vector at the starting point is unique. Thus, as in the Euclidean case, this second way of describing a symmetry operation contains as much information as the first one. This alternative is perhaps not so useful if we are interested in the image of one point only, but it is very practical when considering a large number of points, as in a lattice or a crystal structure. Moreover, it is also particularly user-friendly because symmetry operations are represented by linear maps.

Let us illustrate this by the example of a two-dimensional modulated square lattice $\tilde{\Lambda}^{\square}=\left\{H(a \lambda) \mid \lambda \in \mathbb{Z}^{2}\right\}$, where $a \in \mathbb{R}_{+}$. As seen before, the corresponding non-modulated lattice has a fourfold point of coordinates $(a / 2 ; a / 2)$. Since a rotation $\phi$ of $\pi / 2$ around this point is given in coordinates by relation (14), $\tilde{\phi}=H \circ \phi \circ H^{-1}$ is a symmetry operation of $\tilde{\Lambda}^{\square}$. Let $H(\tilde{q})$ be an 'origin point', with coordinates $H\left(u_{\tilde{q}}\right)$. A curve, parameterized between 0 and 1 , linking this point to any node of $\tilde{\Lambda}^{\square}$, may be written as $t \mapsto H\left(u_{\tilde{q}}+\left(a \lambda-u_{\tilde{q}}\right) t\right)=c(t)$. The components of its tangent vector at $\tilde{q}$ are 


$$
v=\left(\begin{array}{cc}
\omega_{1}^{1}(\tilde{q}) & \omega_{2}^{1}(\tilde{q}) \\
\omega_{1}^{2}(\tilde{q}) & \omega_{2}^{2}(\tilde{q})
\end{array}\right)\left(\begin{array}{c}
a \lambda^{1}-u_{\tilde{q}}^{1} \\
a \lambda^{2}-u_{\tilde{q}}^{2}
\end{array}\right)
$$

where $\omega_{j}^{i}(\tilde{q})=\left.\left(\partial h^{i} / \partial u^{j}\right)\right|_{\tilde{q}}, 1 \leq i, j \leq 2$, are the components of the matrix $\Omega(\tilde{q})$, which represents $\mathrm{d} H_{\tilde{q}}$. By applying $H \circ \phi \circ H^{-1}$ to $H(\tilde{q})$ [with coordinates $H\left(u_{\tilde{q}}\right)$ ], we obtain its image $H\left(\tilde{q}^{\prime}\right)=H(\phi(\tilde{q}))$ which can be considered as a new 'origin point'. Then, taking the matrix part of $\phi$, sandwiching it between $\Omega(\phi(\tilde{q}))$ and $\Omega(\tilde{q})^{-1}$, we obtain the matrix product

(1)

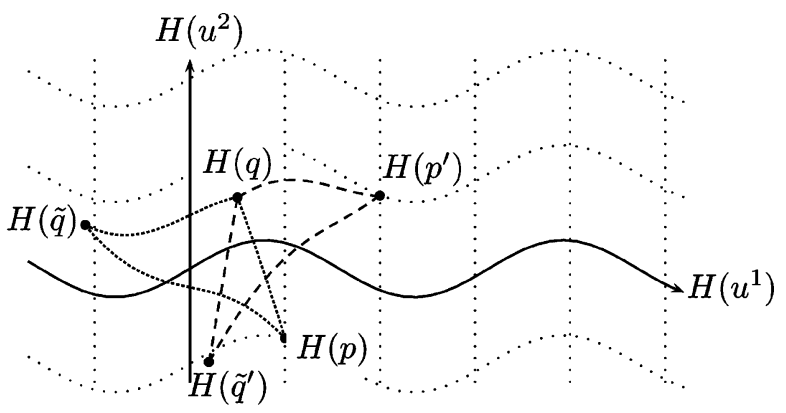

$(2)$

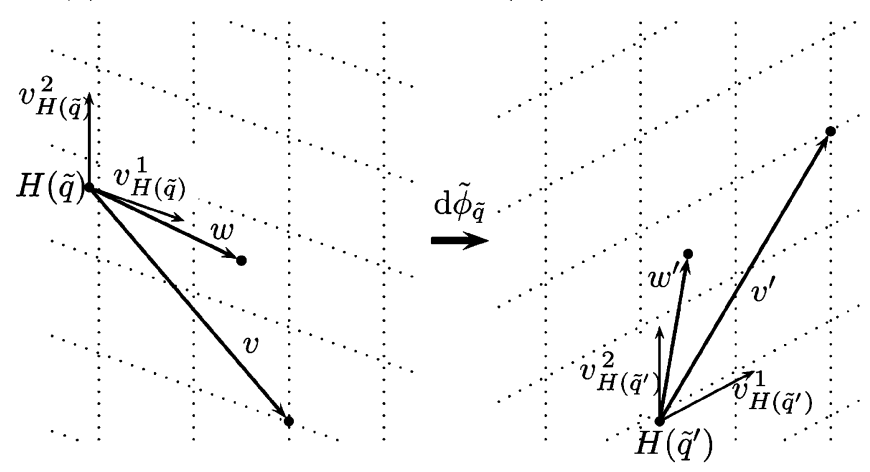

(3)

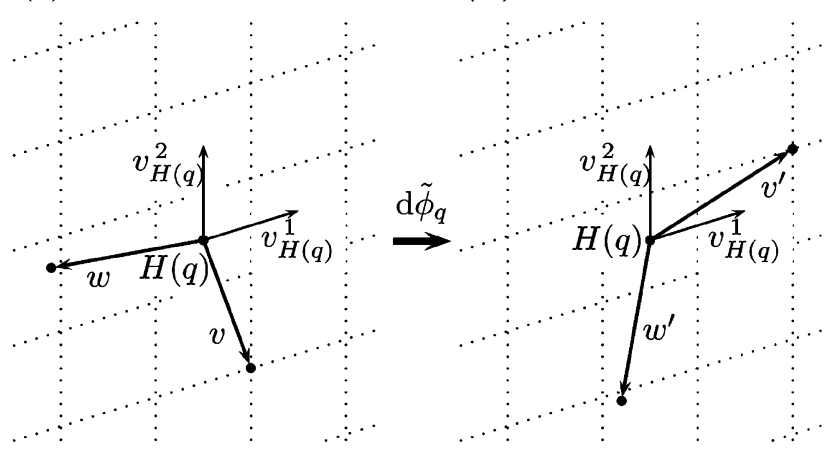

Figure 5

Representations of a symmetry operation in a two-dimensional modulated square lattice. As in the Euclidean example, (1) shows the manifold representation of the 'fourfold points' $H \circ \phi \circ H^{-1}$. The two 'triangles' $H(q), H(\tilde{q}), H(p)$ and $H(q), H\left(\tilde{q}^{\prime}\right), H\left(p^{\prime}\right)$, the edges of which are not straight lines but curves, are also represented. (2) and $\left(2^{\prime}\right)$ show the tangent-space representation of $\phi$. Note the difference of the shape of the lattice in $\mathrm{T}_{H(\tilde{q})} M$ and $\mathrm{T}_{H\left(\tilde{q}^{\prime}\right)} M$. (3) and (3') illustrate the situation in $\mathrm{T}_{H(q)} M$; as $H(q)$ is a fixed point, the differential map $\mathrm{d} \tilde{\phi}_{q} \doteqdot \mathrm{d} H_{q} \circ \mathrm{d} \phi_{q} \circ\left(\mathrm{d} H_{q}\right)^{-1}$ is an endomorphism of the tangent space at $H(q)$.

$$
\left(\begin{array}{ll}
\omega_{1}^{1}\left(\tilde{q}^{\prime}\right) & \omega_{2}^{1}\left(\tilde{q}^{\prime}\right) \\
\omega_{1}^{2}\left(\tilde{q}^{\prime}\right) & \omega_{2}^{2}\left(\tilde{q}^{\prime}\right)
\end{array}\right)\left(\begin{array}{cc}
0 & -1 \\
1 & 0
\end{array}\right)\left(\begin{array}{ll}
\varpi_{1}^{1}(\tilde{q}) & \varpi_{2}^{1}(\tilde{q}) \\
\varpi_{1}^{2}(\tilde{q}) & \varpi_{2}^{2}(\tilde{q})
\end{array}\right),
$$

where $\varpi_{j}^{i}(\tilde{q}), 1 \leq i, j \leq 2$ are the components of the matrix inverse to $\Omega(\tilde{q})$. This product of matrices transforms the vector $v$, of $\mathrm{T}_{H(\tilde{q})} M$, into the vector

$$
v^{\prime}=\left(\begin{array}{cc}
\omega_{1}^{1}\left(\tilde{q}^{\prime}\right) & \omega_{2}^{1}\left(\tilde{q}^{\prime}\right) \\
\omega_{1}^{2}\left(\tilde{q}^{\prime}\right) & \omega_{2}^{2}\left(\tilde{q}^{\prime}\right)
\end{array}\right)\left(\begin{array}{c}
-a \lambda^{2}+u_{\tilde{q}}^{2} \\
a \lambda^{1}-u_{\tilde{q}}^{1}
\end{array}\right),
$$

of $\mathrm{T}_{H\left(\tilde{q}^{\prime}\right)} M$. Moreover, this vector is the tangent vector at $H\left(\tilde{q}^{\prime}\right)=H(\phi(\tilde{q}))$ of the curve parameterized between 0 and 1 linking $H\left(\tilde{q}^{\prime}\right)$ to the image of the nodes of the modulated lattice (see Fig. 5).

Following this last example and our previous calculations, it is apparent that all the concepts introduced in the Euclidean case may be extended to modulated structures. We can therefore give the following general definitions.

(1) The $n$-dimensional point space is an $n$-dimensional (flat) manifold parameterized by a one-to-one map $H: \mathbb{R}^{n} \rightarrow \mathbb{R}^{n}$. If $H$ is the identity map, this manifold is just $\mathbb{R}^{n}$ endowed with the natural coordinate system; hence it corresponds to the $n$ dimensional Euclidean space. When $H$ is a periodic deformation of $\mathbb{R}^{n}$, the manifold corresponds to an $n$-dimensional modulated space in which modulated structures are described. This manifold corresponds essentially to $\mathbb{R}^{n}$; it is just endowed with a curved coordinate system (and not the natural one).

(2) The associated vector space is a tangent space of the manifold (the point space) at a particular point. Thus, as there are an infinity of tangent spaces, there is not just one associated vector space but an infinity. All these tangent spaces are isomorphic. In the Euclidean manifold, they just happen to be 'geometrically equivalent' in the sense that they all have the same canonical (orthonormal) basis. (This is probably the reason why they are often merged and why one speaks about one and not several associated vector spaces.)

(3) A space-group operation is a map which acts in the point space, in the manifold, in which a (modulated) crystal structure is described. In coordinates, such an operation may be written as $H \circ \phi \circ H^{-1}$, where $H$ is the parameterization of the manifold and $\phi$ an element of a discrete subgroup of the isometry group of the Euclidean space. This subgroup contains all symmetry operations leaving the average crystal structure invariant. Thus, $H \circ \phi \circ H^{-1}$ leaves the corresponding modulated structure invariant as well. Note that as all $\phi$ form a group, the set of all $H \circ \phi \circ H^{-1}$ also has the structure of a group. In the case where $H$ is the identity map, the space (hence the crystal) is not modulated and $H \circ \phi \circ H^{-1}=\phi$.

(4) A point-group operation is a linear map between two tangent spaces of the manifold in which the crystal structure exists. It corresponds to the differential map of the spacegroup operation $\phi$. In matrix notation, such an operation may be written as $\Omega\left(q^{\prime}\right) F \Omega(q)^{-1}$, where $\Omega$ and $F$ are the matrix representations of the differential maps $\mathrm{d} H$ and $\mathrm{d} \phi$, respectively, and $q^{\prime}=\phi(q)$. As all elements $\phi$ which leave an average structure invariant form a group, so do the matrices $F$ representing $\mathrm{d} \phi$, hence $\Omega\left(q^{\prime}\right) F \Omega(q)^{-1}$. In the case where $H$ is the identity map, we have simply $\Omega\left(q^{\prime}\right) F \Omega(q)^{-1}=F$. 
Note once again that a symmetry operation in the tangentspace representation gives as much information as in the manifold representation. A point-group operation is usually not an endomorphism of a vector space (as is the case in the traditional point of view), but is a linear map between two tangent spaces. Thus, the translation part does not vanish; it appears in the image of a chosen tangent point.

A last remark concerning $H$. We have always considered the map $H$ to be smooth everywhere. If we want to describe all modulated structures, we also need to include the case where $H$ is smooth piecewise only. In such a case, there exist some points where the derivative of $H$ does not exist. However, all these points form a set of measure zero and it is thus always possible to find points where the tangent space exists. As the set of symmetry operations of a crystal structure is countable, one can always find two points where tangent spaces do exist, the second point being the image of the first one through the symmetry operation.

\section{One-dimensional modulated structure}

In order to illustrate the formalism developed in this article and compare it with the superspace model, nothing is better than to treat a concrete example of a modulated crystal. A one-dimensional structure will be considered, as the visualization of figures becomes difficult in three dimensions and impossible in more than three. Let $\mathcal{S}$ be a one-dimensional non-modulated structure with linear group $p \overline{1}$, containing three atoms in a unit cell. According to the symmetry group,

(1)

(2)

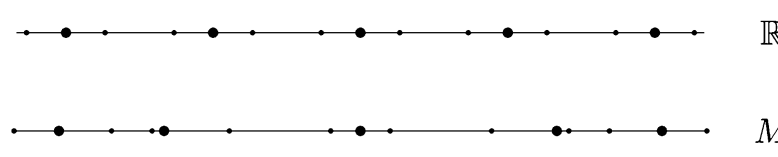

(3)

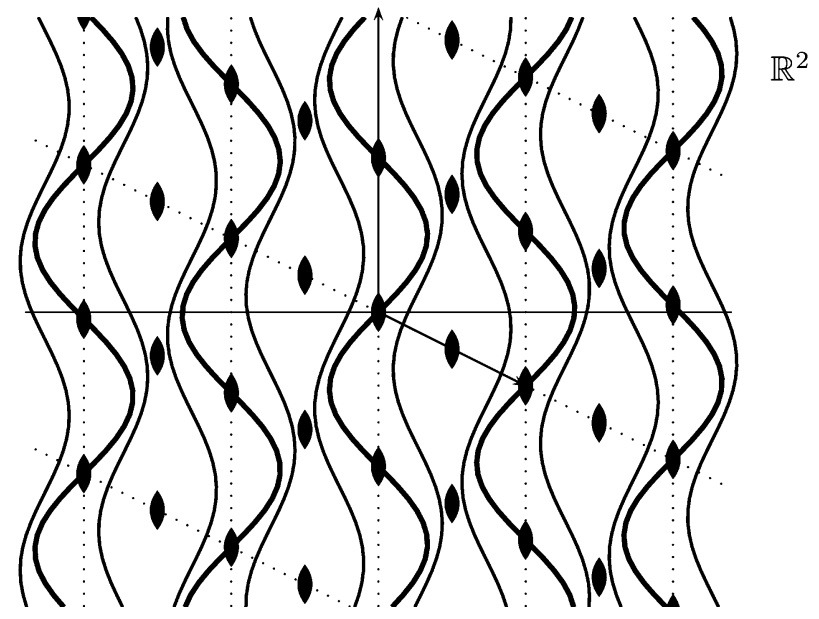

Figure 6

Superspace representation of a one-dimensionally modulated structure. (1) represents the average one-dimensional structure, (2) the corresponding modulated structure and (3) shows the extension of the modulated structure into two-dimensional superspace. Atoms are no longer points, but lines. Twofold points appear in the two-dimensional pattern. two of the three atoms are identical, one atom $\alpha$ and two atoms $\beta$ are located in a unit cell. Their positions are

$$
u_{\alpha}=a \lambda \quad \text { and } \quad u_{\beta}=a \lambda \pm b, \quad \lambda \in \mathbb{Z},
$$

where $a \in \mathbb{R}_{+}$is the unit-cell parameter and $0<b<\frac{1}{2} a$. Consider further a sinusoidal modulation, such that the atoms $\alpha$ and $\beta$ lie on the positions

$$
\begin{aligned}
x_{\alpha} & =a \lambda+A_{\alpha} \sin (\xi a \lambda), \\
x_{\beta_{1}} & =a \lambda+b+A_{\beta} \sin [\xi(a \lambda+b)+\varphi], \\
x_{\beta_{2}} & =a \lambda-b+A_{\beta} \sin [\xi(a \lambda-b)-\varphi],
\end{aligned}
$$

where $\xi, \varphi, A_{\alpha}, A_{\beta} \in \mathbb{R}$ (these parameters may in fact be chosen positive).

In the superspace formalism, one extends the modulated structure into a two-dimensional vector space endowed with the (non-orthonormal) basis $\left\{{ }_{2} a_{1} ;{ }_{2} a_{2}\right\}$. The components of these two vectors, with respect to an orthonormal basis, are

$$
{ }_{2} a_{1}=\left(\begin{array}{c}
a \\
-\xi a / \xi_{\perp}
\end{array}\right), \quad{ }_{2} a_{2}=\left(\begin{array}{c}
0 \\
-1 / \xi_{\perp}
\end{array}\right),
$$

where $\xi_{\perp}$ is an arbitrary constant. The first component of ${ }_{2} a_{1}$ is the lattice parameter of the average structure in the external space, whereas the second one is $\xi a$ times the component of ${ }_{2} a_{2}$ in the internal space (which is perpendicular to the external space). The two-dimensional structure is shown in Fig. 6. The pattern has twofold rotation symmetry around points of coordinates $\left(n^{1} / 2\right){ }_{2} a_{1}+\left(n^{2} / 2\right){ }_{2} a_{2}$, where $n^{1}, n^{2} \in \mathbb{Z}$. The matrix part of these symmetry operations is

$$
\left(\begin{array}{c|c}
-1 & 0 \\
\hline 0 & -1
\end{array}\right)
$$

the element $(1 ; 1)$ of which corresponds to the inversion operation of the average structure.

In the formalism based on differential geometry, the whole one-dimensional space is modulated (distorted) by a map and the modulated structure is 'periodic' in this distorted space. Let us first build this map, using the bump function

$$
u \mapsto \varrho_{s}(u)=\left\{\begin{array}{cl}
\exp \left\{1-\left[s^{2} /\left(s^{2}-u^{2}\right)\right]\right\} & \text { when }|u|<s \\
0 & \text { when }|u| \geq s,
\end{array}\right.
$$

where $s \in \mathbb{R}_{+}$. It is smooth, even, and reaches its maximum value 1 at $u=0$. Let us take this function, fix $s=\frac{1}{2} b$, and shift it in such a way as to have the maximum at the position $u_{\beta}$ of one of the $\beta$ atoms (in the average structure). We obtain the following function $u \mapsto \varrho\left(u-u_{\beta}\right)$, where

$$
\begin{aligned}
& \varrho_{b / 2}\left(u-u_{\beta}\right) \\
& \quad=\left\{\begin{array}{cc}
\exp \left\{1-\left\{b^{2} /\left[b^{2}-4\left(u-u_{\beta}\right)^{2}\right]\right\},\right. & \left|u-u_{\beta}\right|<b / 2 \\
0, & \left|u-u_{\beta}\right| \geq b / 2 .
\end{array}\right.
\end{aligned}
$$

With this tool, it is easy to build the one-to-one modulation map $h: \mathbb{R} \rightarrow \mathbb{R}$. It is simply given by

$$
\begin{aligned}
u \mapsto h(u)= & \left(1-\varrho_{b / 2}\left(u-u_{\beta_{1}}\right)-\varrho_{b / 2}\left(u-u_{\beta_{2}}\right)\right) f(u) \\
& +\varrho_{b / 2}\left(u-u_{\beta_{1}}\right) g(u)+\varrho_{b / 2}\left(u-u_{\beta_{2}}\right) \bar{g}(u),
\end{aligned}
$$


where

$$
\begin{aligned}
& f(u)=u+A \sin (\xi u), \\
& g(u)=u+B \sin (\xi u+\varphi), \\
& \bar{g}(u)=u+B \sin (\xi u-\varphi) ;
\end{aligned}
$$

$u_{\beta_{1}}$ and $u_{\beta_{2}}$ are the positions of the two atoms $\beta$. When $u=u_{\alpha}$, we see that $h\left(u_{\alpha}\right)=x_{\alpha}$, and when $u=u_{\beta_{i}}$, we have $h\left(u_{\beta_{i}}\right)=x_{\beta_{i}}, i=1,2$. Through $h$, the image of any point $p$ with coordinate $u_{p}$, for instance such that $a-b<u_{p}<a$, is

$$
h\left(u_{p}\right)=\left(1-\varrho_{b / 2}\left(u_{p}-u_{\beta_{2}}\right)\right) f\left(u_{p}\right)+\varrho_{b / 2}\left(u_{p}-u_{\beta_{2}}\right) \bar{g}\left(u_{p}\right)
$$

and the differential map of $h$ at this point is

$$
\begin{aligned}
\mathrm{d} h_{p}= & -\mathrm{d} \varrho_{b / 2, p} f\left(u_{p}\right)+\left[1-\varrho_{b / 2}\left(u_{p}-u_{\beta_{2}}\right)\right] \mathrm{d} f_{p} \\
& +\mathrm{d} \varrho_{b / 2, p} \bar{g}\left(u_{p}\right)+\varrho_{b / 2}\left(u_{p}-u_{\beta_{2}}\right) \mathrm{d} \bar{g}_{p},
\end{aligned}
$$

where

$$
\begin{aligned}
\mathrm{d} \varrho_{b / 2, p} & =-\frac{8 b^{2}\left(u_{p}-u_{\beta_{2}}\right)}{b^{2}-4\left(u_{p}-u_{\beta_{2}}\right)^{2}} \exp \left[1-\frac{b^{2}}{b^{2}-4\left(u_{p}-u_{\beta_{2}}\right)^{2}}\right], \\
\mathrm{d} f_{p} & =1+\xi A \cos \left(\xi u_{p}\right), \\
\mathrm{d} g_{p} & =1+\xi B \cos \left(\xi u_{p}-\varphi\right) .
\end{aligned}
$$

The positions of the $\alpha$ and $\beta$ atoms of our one-dimensional modulated structure in the tangent space at $h(p)$ are then

$$
\begin{aligned}
& v_{\alpha}=\Omega(p)\left(a \lambda-u_{p}\right), \\
& v_{\beta}=\Omega(p)\left(a \lambda \pm b-u_{p}\right),
\end{aligned}
$$

where $\Omega(p)$ is the $(1 \times 1)$ matrix representing $\mathrm{d} h_{p}$; it is in fact just a number, which provides a kind of scale in tangent space. Thus, the structure in the tangent space has vector periodicity, as it has the structure of a $\mathbb{Z}$-module.

Let us consider the inversion operation, the symmetry element (the fixed point) of which in the average structure lies

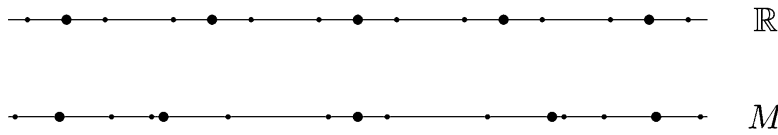

(3)

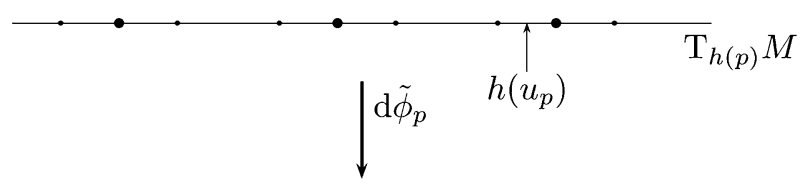

(4)

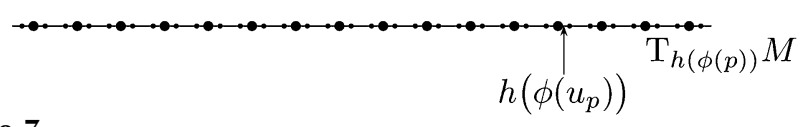

Figure 7

Representation of a one-dimensional modulated structure in tangent space. As in Fig. 6, (1) and (2) show the average and modulated structure, respectively, and (3) shows the representation of the modulated structure in the tangent space at $h(p)$. After the application of the differential map $\mathrm{d} \tilde{\phi}_{p}$ (the 'inversion' map), which carries vectors of the tangent space at $h(p)$ to vectors of the tangent space at $\tilde{\phi}(h(p))=h(\phi(p))$, we obtain (4), which corresponds exactly to the representation of the modulated structure in the tangent space at $\tilde{\phi}(h(p))$. on $\frac{1}{2} a \lambda, \lambda \in \mathbb{Z}$. Let us consider the fixed point localized at $a$. The corresponding operation $\phi$ may be written in coordinates as $u \mapsto \phi(u)=-u+2 a$. In our modulated structure, this operation acts on points of the modulated space as $h(u) \mapsto \tilde{\phi}(h(u))=h(\phi(u))=h(-u+2 a)$, where $\tilde{\phi}=h \circ \phi \circ h^{-1}$. The point $h(a)$ is a fixed point of the operation and may be called a symmetry element as well.

As seen before, this symmetry operation also has a tangentspace representation. The 'origin' point $h\left(u_{p}\right)$ is transferred to a new 'origin' point $h\left(\phi\left(u_{p}\right)\right)$ through $\tilde{\phi}$ and

$$
\mathrm{d} \tilde{\phi}_{p}: \mathrm{T}_{h(p)} M \rightarrow \mathrm{T}_{h\left(\phi\left(u_{p}\right)\right)} M
$$

carries tangent vectors at $h(p)$ into tangent vectors at $h(\phi(p))$ (note that $M$ is mostly $\mathbb{R}$, it is just parameterized by the map $h)$. Thus, the image of the vectors $v_{\alpha}$ and $v_{\beta}$ through $\mathrm{d} \tilde{\phi}_{p}$ is

$$
\begin{aligned}
v_{\alpha}^{\prime} & =\mathrm{d} \tilde{\phi}_{p}\left(v_{\alpha}\right)=\Omega(\phi(p)) F \Omega(p)^{-1} \Omega(p)\left(a \lambda-u_{p}\right) \\
& =\Omega(\phi(p))\left(-a \lambda+u_{p}\right), \\
v_{\beta}^{\prime} & =\mathrm{d} \tilde{\phi}_{p}\left(v_{\beta}\right)=\Omega(\phi(p)) F \Omega(p)^{-1} \Omega(p)\left(a \lambda \pm b-u_{p}\right) \\
& =\Omega(\phi(p))\left(-a \lambda \mp b+u_{p}\right),
\end{aligned}
$$

where $\Omega(\phi(p))$ is the $(1 \times 1)$ matrix representing $\mathrm{d} h_{\phi(p)}$ and $F=-1$ is the $(1 \times 1)$ matrix representing $\mathrm{d} \phi$, the differential map of the (Euclidean) inversion operation $\phi$ (see Fig. 7). These vectors correspond exactly to the points in the manifold obtained by applying $\tilde{\phi}$ to the atomic positions in the modulated structure. Notice that in the case where $u_{p}=a$, $\tilde{\phi}\left(h\left(u_{p}\right)\right)=h\left(u_{p}\right)$ and $\mathrm{d} \tilde{\phi}_{p}$ is an endomorphism of the tangent space at $h(p)$.

This example illustrates well that the symmetry of modulated structures can be described without adding dimensions, i.e. by remaining in the basic space. The notions of spacegroup and point-group operations hold for non-distorted as well as for modulated crystals, without considering an additional dimension. In the formalism developed in this article, a symmetry operation is effectively no longer represented by an affine map. By using the tangent spaces, it can, however, always be represented by a linear map. Another difference between the superspace formalism and the model developed in this article is that the first imposes restrictions on the shape of the modulation and the modulation wavevector, whereas the second does not. Indeed, in the previous one-dimensional example, the pattern in Fig. 6 can be left invariant under rotations of angle $\pi$ only because the sinusoidal function is also left invariant under the same rotations. With the model based on differential geometry, the modulation can have any shape. Typically, the modulation applied to the square lattice in Fig. 5 is allowed in the formalism developed in this article, whereas it is forbidden in the superspace model; indeed, as the average square lattice is left invariant under a rotation of angle $\pi / 2$, a modulation along one axis would necessarily imply a same modulation along the second one.

There is a priori not only one possible construction of the parameterization $H$. Indeed, one is ultimately interested in the precise shape of $H$ only at the (displaced) positions of the 
centres of the atoms in a unit cell; between the atoms, the shape of $H$ seems less important.

The modulation of each atom is a periodic wavefunction of the position. The connection between each of these functions can be obtained by using smooth homotopy; this is what has been done in the previous one-dimensional example: from the atom $\alpha$ to the atoms $\beta$ the modulation of $\alpha$ smoothly vanishes and that of $\beta$ smoothly appears. The smoothness, which is guaranteed by the bump function (used in the example), ascertains that the parameterization $H$ is as smooth as possible, hence that $M$ is a manifold. To see more concretely how it works, we take the example of a two-dimensional structure containing three atoms $\alpha, \beta$ and $\gamma$, each of them being modulated by a different function $H_{\alpha}, H_{\beta}$ and $H_{\gamma}$. Let us consider the coordinate system $x=\left(x^{1} ; x^{2}\right)$ and suppose that the positions of the atoms in the average structure are $x_{\alpha}=\left(x_{\alpha}^{1} ; x_{\alpha}^{2}\right), x_{\beta}=\left(x_{\beta}^{1} ; x_{\beta}^{2}\right)$ and $x_{\gamma}=\left(x_{\gamma}{ }^{1} ; x_{\gamma}{ }^{2}\right)$. In the modulated structure, the positions of the atoms are

$$
\begin{aligned}
& \left(h_{\alpha}^{1}\left(x_{\alpha}\right) ; h_{\alpha}^{2}\left(x_{\alpha}\right)\right)=\left(x_{\alpha}^{1}+\tilde{h}_{\alpha}^{1}\left(\xi_{1} x_{\alpha}^{1}\right) ; x_{\alpha}^{2}+\tilde{h}_{\alpha}^{2}\left(\xi_{1} x_{\alpha}^{1}\right)\right) \\
& \left(h_{\beta}^{1}\left(x_{\beta}\right) ; h_{\beta}^{2}\left(x_{\beta}\right)\right)=\left(x_{\beta}^{1}+\tilde{h}_{\beta}^{1}\left(\xi_{1} x_{\beta}^{1}\right) ; x_{\beta}^{2}+\tilde{h}_{\beta}^{2}\left(\xi_{1} x_{\beta}^{1}\right)\right) \\
& \left(h_{\gamma}{ }^{1}\left(x_{\gamma}\right) ; h_{\gamma}{ }^{2}\left(x_{\gamma}\right)\right)=\left(x_{\gamma}{ }^{1}+\tilde{h}_{\gamma}{ }^{1}\left(\xi_{1} x_{\gamma}^{1}\right) ; x_{\gamma}{ }^{2}+\tilde{h}_{\gamma}{ }^{2}\left(\xi_{1} x_{\gamma}{ }^{1}\right)\right),
\end{aligned}
$$

where $H_{i}=\left(h_{i}{ }^{1} ; h_{i}{ }^{2}\right)$ and $\tilde{H}_{i} \doteqdot\left(\tilde{h}_{i}{ }^{1} ; \tilde{h}_{i}{ }^{2}\right), i=\alpha, \beta, \gamma, \tilde{H}_{i}$ being a periodic wavefunction of the position. Whereas all the atoms of a unit cell in a modulated structure have a different modulation, the wavelength of all these functions is the same. A parameterization $H$ may be obtained by smoothly distorting one of the $H_{i}$ to another one. Without restriction, one can suppose that $x_{\alpha}^{2}<x_{\beta}^{2}<x_{\gamma}^{2}$. Then, one needs to find a smooth
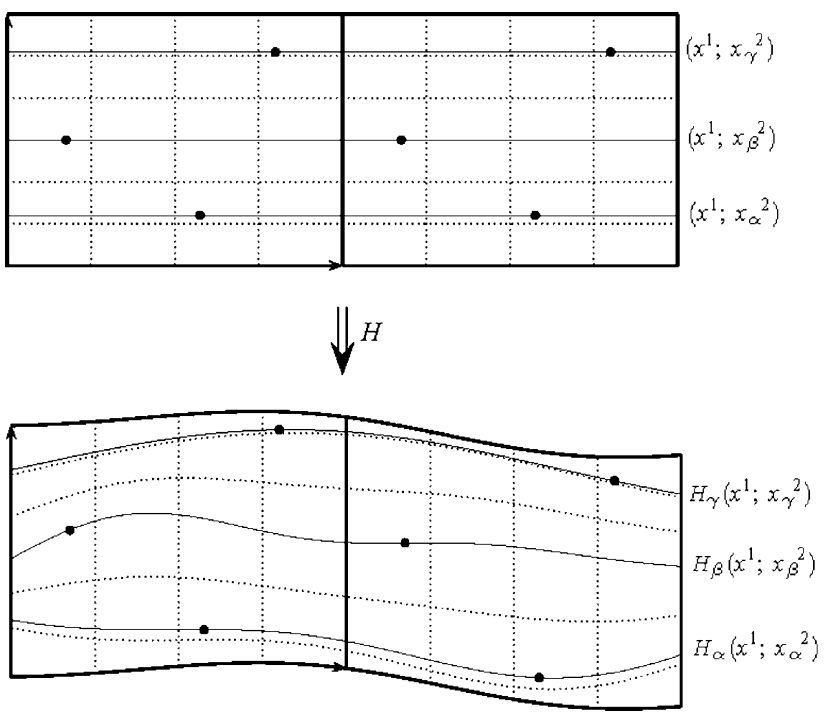

\section{Figure 8}

Representation of two unit cells of a modulated structure obtained by a deformation through the parameterization $H$ of two unit cells of the corresponding average structure. The map $H$ is obtained from the modulation functions $H_{\alpha}, H_{\beta}$ and $H_{\gamma}$ of the atoms $\alpha, \beta$ and $\gamma$, respectively, by using the concept of smooth homotopy. The displacement of the atoms may be obtained by taking the difference between the positions in the corresponding modulated and non-modulated cells. path from $H_{\alpha}\left(x^{1} ; x_{\alpha}^{2}\right)$ to $H_{\beta}\left(x^{1} ; x_{\beta}^{2}\right)$, another one from $H_{\beta}\left(x^{1} ; x_{\beta}^{2}\right)$ to $H_{\gamma}\left(x^{1} ; x_{\gamma}^{2}\right)$, and finally, by periodicity, another one from $H_{\gamma}\left(x^{1} ; x_{\gamma}^{2}\right)$ to $H_{\alpha}\left(x^{1} ; x_{\alpha}^{2}+1\right)$. Using the bump function $\varrho$, we write

$$
H\left(x^{1} ; x^{2}\right)=\left(h^{1}\left(x^{1} ; x^{2}\right) ; h^{2}\left(x^{1} ; x^{2}\right)\right)
$$

where

$$
\begin{aligned}
h^{j}\left(x^{1} ; x^{2}\right)= & x^{j}+\left(1-\varrho_{s_{\beta}}\left(x^{2}-x_{\beta}^{2}\right)-\varrho_{s_{\gamma}}\left(x^{2}-x_{\gamma}^{2}\right)\right) \tilde{h}_{\alpha}^{j}\left(\xi_{1} x^{1}\right) \\
& +\varrho_{s_{\beta}}\left(x^{2}-x_{\beta}^{2}\right) \tilde{h}_{\beta}{ }^{j}\left(\xi_{1} x^{1}\right)+\varrho_{s_{\gamma}}\left(x^{2}-x_{\gamma}^{2}\right) \tilde{h}_{\gamma}{ }^{j}\left(\xi_{1} x^{1}\right),
\end{aligned}
$$

with $s_{\beta}+s_{\gamma} \leq x_{\gamma}^{2}-x_{\beta}^{2}, s_{\beta} \leq x_{\beta}^{2}-x_{\alpha}^{2}$ and $s_{\gamma} \leq x_{\alpha}^{2}+1-x_{\gamma}^{2}$, in order that the bumps have an appropriate width. The representation of the parameterization $H$ is shown in Fig. 8 .

If we are interested in the positions of the centres of the atoms only, the parameterization $H$ is not unique, as there are many smooth paths to go from one modulation function to another one. In fact, if we consider the electron density of the whole structure and could compare it with the electron density of the corresponding average structure, we might obtain a unique expression for the parameterization $H$, as an electron density is a function which is non-negative not only close to the centres of atoms but everywhere.

\section{Conclusion and perspectives}

This analysis does not aim at completely changing all practical calculations. Rather we have shown that there exist some mathematical tools particularly suited for the description of certain physical phenomena. Differential geometry offers a suitable framework for the description of crystals. Its tools and spaces turn out to be tailored to demonstrating the same concepts as those used by crystallographers in quite a natural way. In particular, we see that there are two equivalent ways of finding the image of a point through a symmetry operation, one directly in the manifold, the second one using tangent spaces.

One might criticize the fact that the position of atoms (of a crystal structure) in the tangent space does not always correspond to their real position in the structure. This is right, but a one-to-one correspondence between vectors in the tangent space and points in the manifold always exists. Thus, as soon as the image of a vector (representing a point) in the tangent space is given, the corresponding image in the manifold can be obtained. Moreover, the representation of a modulated structure in superspace does not correspond to physical reality either; the atoms are not points but lines, surfaces or volumes, and if the real three-dimensional structure has to be derived, a section of the superspace structure needs to be considered. Thus, some effort is required in any case; either one needs additional dimensions or one considers the tangent-space representation.

It would seem from the literature dedicated to modulated structures that any symmetry operation must be represented by a matrix and a translational part, as if this were a 'golden rule'. Accepting this effectively requires the definition of a higher-dimensional space in which an appropriate higher- 
dimensional periodic structure is defined. But the rigour required for the definition of fundamental concepts in crystallography handsomely pays back with a user-friendly representation of symmetry operations, like linear maps between tangent spaces, without using a higher-dimensional space.

Note that other models for describing the symmetry of modulated structures, without adding dimensions, have been proposed in the past, notably by Perez-Mato and co-workers in 1983 (Perez-Mato et al., 1984), and Mermin and co-workers in 1995 (Dräger \& Mermin, 1996). Be they based on the invariance of the Landau free energy or on gauge transformations, both formalisms treat modulated structures in reciprocal space, whereas the description of symmetry operations presented here takes place in direct space.

Differential geometry appears to be an elegant frame for the description of symmetry operations. The tangent-space representation of such an operation is particularly practical. The next step will be to solve the transition from one tangent space to another one and to obtain a structure in any tangent space once it is known from another one. This requires the introduction of an equivalence relation on the tangent bundle of a manifold.

PhK warmly acknowledges Professor Stephen Hyde for his cordial welcome to the Department of Applied Mathematics of the Australian National University in Canberra, and for interesting and very fruitful discussions. Many thanks are due to him and also to members of his group (especially Stuart Ramsden, Myfanwy Evans and Vanessa Robins) for an introduction to their field of research. The support for this work provided by the Swiss National Science Foundation (grant 20-113605) is gratefully acknowledged.

\section{References}

Burzlaff, H. \& Zimmermann, H. (1977). Symmetrielehre, ch. III. Stuttgart: Georg Thieme Verlag.

Das, A. (1993). The Special Theory of Relativity, ch. 2, pp. 41-43. New York: Springer-Verlag.

Dräger, J. \& Mermin, N. D. (1996). Phys. Rev. Lett. 76, 1489-1492.

Engel, P. (1986). Geometric Crystallography, ch. 1, pp. 1-12. Dordrecht: D. Reidel Publishing Company.

Janssen, T., Chapuis, G. \& de Boissieu, M. (2007). Aperiodic Crystals. Oxford University Press.

Janssen, T., Janner, A., Looijenga-Vos, A. \& de Wolff, P. M. (2002). Basic Structural Features: Incommensurate and Commensurate Modulated Structures. International Tables for Crystallography, Vol. C, ch. 9.8, pp. 907-937. Dordrecht: Kluwer Academic Publishers.

Kleman, M. \& Friedel, F. (2008). Rev. Mod. Phys. 80, 61-115.

O'Neill, B. (1983). Semi-Riemannian Geometry. London: Academic Press.

Opęchowski, W. (1986). Crystallographic and Metacrystallographic Groups, ch. 4, pp. 537-558. Amsterdam: North-Holland.

Perez-Mato, J. M., Madariaga, G. \& Tello, M. J. (1984). Phys. Rev. B, 30, 1534-1543.

Schwarzenbach, D. (1993). Cristallographie, ch. 2, pp. 27-34. Lausanne: Presses Polytechniques et Universitaires Romandes.

Smaalen, S. van (1995). Crystallogr. Rev. 4, 79-202.

Smaalen, S. van (2007). Incommensurate Crystallography. Oxford University Press.

Steurer, W. \& Haibach, T. (2001). Reciprocal-Space Images of Aperiodic Crystals. International Tables for Crystallography, Vol. B, ch. 4.6, pp. 486-532. Dordrecht: Kluwer Academic Publishers.

Wondratschek, H. (2002). Introduction to Space-group Symmetry: Basic Concepts. International Tables for Crystallography, Vol. A, ch. 8.1, pp. 720-721. Dordrecht: Kluwer Academic Publishers. 